\title{
Role of microstructure on fracture of dentin
}

\author{
Bingbing $\mathrm{An}^{\mathrm{a}, \mathrm{b}, *}, \mathrm{H}$. Daniel Wagner ${ }^{\mathrm{a}}$ \\ ${ }^{\mathrm{a}}$ Department of Materials and Interfaces, Weizmann Institute of Science, Rehovot \\ 76100, Israel \\ ${ }^{\mathrm{b}}$ Department of Mechanics, Shanghai University, Shanghai 200444, People's Republic \\ of China
}

*Corresponding author

Bingbing An, Ph.D.

Department of Materials and Interfaces

Weizmann Institute of Science

Rehovot 76100, Israel

Department of Mechanics

Shanghai University

Shanghai 200444, People's Republic of China

Email: bingbing.an@weizmann.ac.il, anbingbing@shu.edu.cn 
Abstract Dentin possesses unique hierarchical structure, which has a significant influence on the mechanical properties. Understanding the relationship between structure and mechanical properties of dentin is essential for preventing and curing oral diseases, as well as, potentially for developing man-made engineering materials with superior mechanical performance. In this study, the effect of the two-layered structure, where hard peritubular dentin (PTD)containing dentin tubules are embedded in soft intertubular dentin (ITD), on the fracture behavior of dentin is investigated. A numerical model is developed, in which PTD cracking, ITD cracking and the debonding of the interface between PTD and ITD are all taken into account. Numerical simulations reveal that PTD fracture and interface debonding are the major failure mechanisms, which are consistent with experimental observation. It is identified that the cohesive strength and critical separation of interface are the key parameters controlling which of the mechanisms is active. The low cohesive strength of interface and small critical separation of interface can lead to interface debonding, while the large cohesive strength and critical separation give rise to PTD fracture. In addition, it is found that large volume fraction of dentin tubules and small volume fraction of PTD can enhance the toughness of dentin, which provides a new insight into the degraded mechanical properties of old dentin.

Keywords: Dentin; fracture; failure mechanism; two-layered structure; toughness 


\section{Introduction}

Dentin, the major component of tooth, possesses complex hierarchical structure. At the nanoscale, dentin is a biocomposite consisting of staggered arrays of mineral crystals, embedded in a protein matrix (Gao et al., 2003; Bar-On and Wagner, 2012a; An et al., 2014). On the micron length scale, a remarkable feature of dentin is the existence of dentin tubules, which traverse dentin and display a range of diameters (Deymier-Black et al., 2010; Zaslansky et al., 2010;Ivancik and Arola, 2013). The dentin tubules near the pulp have a diameter of $2.5 \mu \mathrm{m}$, while the diameter of the dentin closer to enamel is reduced to approximately $1 \mu \mathrm{m}$ (Stock et al., 2011; Ryou et al., 2012). The dentin tubules are embodied by the mineral-rich peritubular dentin (PTD), which are further wrapped by the protein-rich soft intertubular dentin (ITD), forming a unique two-layered structure (Bertassoni et al., 2012; An and Zhang, 2015).

This unique hierarchical structure has a profound effect on the mechanical properties of dentin. A study by Iwamoto and Ruse (2003) revealed that the fracture toughness in the direction perpendicular to dentin tubules is significantly lower than that in the direction parallel to dentin tubules, which is attributed to the existence of brittle PTD and the arrangement of collagen fibers in the vicinity of dentin tubules. For the cracking along the dentin tubules, crack bridging caused by uncracked ligaments and collagen fibers appears in the crack wake, which alleviates the stress intensity at the crack tip and increases the propensity of crack closure, thereby enhancing fracture toughness. However, these toughening mechanisms are not observed for the crack growth perpendicular to dentin tubules (Nalla et al., 2003). Due 
to the development of crack bridging, the fracture toughness of dentin increases with crack extension, which suppresses crack propagation and mitigates fracture (Koester et al., 2008; Nazari et al., 2009). Ivancik and Arola (2013) found that during crack propagation, a significant degree of microcracking in PTDs occurs, which dissipates energy and contributes to the fracture toughness of dentin. Due to the existence of dentin tubules, dentin also display anisotropic fatigue crack growth behavior (Ivancik et al., 2012). Arola et al (2007) reported that the average Paris law exponent corresponding to the crack growth perpendicular to dentin tubules (transverse direction) is much larger than that for the case of crack path oblique to dentin tubules, indicating that the mechanical properties of dentin in the transverse direction are similar to that of brittle materials. The study by Ivancik et al (2011) identified the influence of the density of dentin tubules; large tubule density gives rise to the high fatigue crack growth, leading to the weak resistance to crack growth. For fatigue crack growth in dentin, besides the toughening mechanisms of crack bridging and microcracking, there exists another important toughening mechanism related to the microstructure. Bajaj et al (2006) observed that PTD pullout takes place, which can dissipate energy effectively, thereby benefiting damage tolerance.

In addition to the effect on fracture behavior, the elastic properties of dentin are also associated with its peculiar hierarchical structure. Han et al (2012) conducted micropillar compression experiment on dentin and found that the elastic modulus of dentin is dependent on the loading direction with respect to the dentin tubules. The high elastic modulus appears when the loading direction is parallel to the dentin 
tubules, whereas the elastic modulus in the direction perpendicular to dentin tubules is low. The study by Bar-On and Wagner (2012a,b) showed that the elastic modulus of dentin is a function of tubule content and ITD modulus.

Despite these efforts to elucidate the relation between structure and property of dentin, there are few studies focusing on the inelastic deformation of dentin. As a matter of fact, understanding of the inelastic deformation mechanisms can lead to a deeper insight into the fracture resistance of dentin, since the capability of materials to undergo large inelastic deformation is an important toughening mechanism. In this regard, Eltit et al (2013) conducted bending experiment on dentin and attempted to identify the nature of the inelastic deformation of dentin. It was found that microcracks develop in the PTD and then propagate through ITD; some microcracks stop at the interface between PTD and ITD, where interfacial debonding seems to be the plausible damage mechanism. Why are the microcracks arrested at the interface? Why does dentin display competing damage mechanisms? Does the two-layered structure play an important role? To address these issues, a good understanding of the relation between the two-layered structure and damage mechanisms of dentin is required. The primary goal of this study is to reveal the role of the two-layered structure on the fracture of dentin. A micromechanical model accounting for ITD cracking, PTD cracking and interfacial debonding is developed. The influence of the mechanical properties of ITD, PTD and interface on the toughness of dentin is elucidated and the key parameters governing the transition in damage mechanisms are identified. 


\section{Method}

A model of a solid with a two-layered composite structure is developed to represent the dentin microstructure (Fig. 1). The PTD containing dentin tubules is modeled as a hollow cylindrical reinforcement with mechanical properties characterized by a linear elastic model; the ITD is modeled as the matrix, the constitutive relation of which is also described by a linear elastic model. In view of the periodic nature of the microstructure, the mechanical properties of the overall microstructure can be obtained by considering the unit cell with dimension of $2 \mathrm{~L} \times 2 \mathrm{~L}$ shown in Fig. 1(b). Owing to the symmetry of the unit cell, only one quarter of the unit cell is modeled. Based on the bending experiment conducted by Eltit et al (2013), where the cracks nucleating in the PTD propagate along the direction perpendicular to the tensile stress, the plane of symmetry of the two-layered structure perpendicular to the applied tensile stress is considered as potential crack plane with a mechanical response characterized by a cohesive zone model, which is represented by the relation between traction and separation (Xu and Needleman, 1994; Moslerand Scheider, 2011; An et al., 2014).The separation characterizes the deformation of interfaces. For a material system composed of two solids separated by an interface $A_{0}$, an upper surface $A^{+}$and a lower surface $A^{-}$, which coincide with the interface $A_{0}$ in the undeformed configuration, will emerge when the system is subjected to external loading. The material point $\boldsymbol{X}$ initially on $A_{0}$ will appear on the surfaces $A^{+}$and $A^{-}$with positions of $\boldsymbol{x}^{+}$and $\boldsymbol{x}^{-}$, respectively. Separation is defined as $\boldsymbol{\delta}=\boldsymbol{x}^{+}-\boldsymbol{x}^{-}$(Xu and Lu, 2013). For the 
fracture of the microstructure of dentin, PTD fracture and the fracture of the interface between PTD and ITD are the major failure mechanisms (Arola and Reprogel, 2005). To examine the competition between interfacial debonding and PTD cracking, the interface between PTD and ITD is also modeled utilizing cohesive zone model.

Since it is known that the shape of the traction-separation curve does not affect the fracture behavior (Tvergaard and Hutchinson, 1992), the simple bilinear cohesive zone model (Ural and Vashishth, 2006, 2007) is employed here. The damage initiation is controlled by the maximum stress criterion,

$$
\max \left\{\frac{\left\langle T_{n}\right\rangle}{\sigma_{n}}, \frac{T_{s}}{\sigma_{s}}, \frac{T_{t}}{\sigma_{t}}\right\}=1
$$

where $T_{n}, T_{\mathrm{s}}$ and $T_{t}$ are the tractions in the normal and tangential directions, respectively; $\sigma_{n}, \sigma_{s}$ and $\sigma_{t}$ are the strengths in the normal and tangential directions, respectively. The symbol<> represents the McCauley bracket, defined as $\langle T n\rangle=T n$ if $T n>0$, and $\langle T n\rangle=0$ if $T n \leq 0$. Crack nucleation and propagation in materials can be considered as a damage evolution process, therefore a scalar damage variable, $D$, with the initial value of 0 , is introduced. With this damage variable, the tractions in the three directions are given by

$$
\begin{gathered}
T_{n}=\left\{\begin{array}{c}
(1-D) \bar{T}_{n}, \text { for } \bar{T}_{n} \geq 0 \\
\bar{T}_{n}, \text { otherwise }
\end{array}\right. \\
T_{s}=(1-D) \bar{T}_{s} \\
T_{t}=(1-D) \bar{T}_{t}
\end{gathered}
$$

where $\bar{T}_{n}, \bar{T}_{s}$ and $\bar{T}_{t}$ are effective tractions in the normal and the two tangential directions, respectively. These effective tractions describe the mechanical properties of undamaged materials and can be written as 


$$
\begin{gathered}
\bar{T}_{n}=\frac{\sigma_{n}}{\delta_{n c}} \delta_{n} \\
\bar{T}_{s}=\frac{\sigma_{s}}{\delta_{s c}} \delta_{s} \\
\bar{T}_{t}=\frac{\sigma_{t}}{\delta_{t c}} \delta_{t}
\end{gathered}
$$

where $\delta_{n c}, \delta_{s c}$ and $\delta_{t c}$ are critical separations in the normal and the two tangential directions, respectively; $\delta_{n}, \delta_{s}$ and $\delta_{t}$ are separations in the normal and the two tangential directions, respectively. In this study, we set $\sigma_{n}=\sigma_{s}=\sigma_{t}=\sigma$ and $\delta_{n c}=\delta_{s c}=\delta_{t c}=\delta_{c}$. The area under the traction-separation curve is fracture energy $\mathrm{G}$, and $G_{n}=G_{s}=G_{t}=$ $G$ is assumed throughout this paper. To characterize the effect of mixed deformation on the evolution of damage variable, an effective separation is introduced,

$$
\hat{\delta}=\sqrt{\left\langle\delta_{n}\right\rangle^{2}+\delta_{s}^{2}+\delta_{t}^{2}}
$$

With this definition, the damage variable can be expressed as

$$
D=\frac{\widehat{\delta}_{f}\left(\widehat{\delta}_{\max }-\widehat{\delta}_{c}\right)}{\widehat{\delta}_{\max }\left(\widehat{\delta}_{f}-\widehat{\delta}_{c}\right)}
$$

where $\hat{\delta}_{\max }$ is the maximum effective separation during the loading process and $\hat{\delta}_{f}$ is the separation corresponding to the complete failure. The relationship between traction and separation is shown in Fig. 1(a).

The plane strain condition is considered and a tensile load in the 2-direction shown in Fig. 1(b) is applied. The constitutive equations, boundary conditions and balance equations constitute a boundary value problem. To solve this problem, a numerical method based on the principle of virtual work is adopted, which takes the form

$$
\begin{array}{r}
\int_{B_{P}} \boldsymbol{\sigma}: \tilde{\boldsymbol{\varepsilon}} d V+\int_{B_{I}} \boldsymbol{\sigma}: \tilde{\boldsymbol{\varepsilon}} d V+\int_{\Gamma_{I}} \boldsymbol{T} \cdot \widetilde{\boldsymbol{\delta}} d S+\int_{\Gamma_{1}} \boldsymbol{T} \cdot \widetilde{\boldsymbol{\delta}} d S+\int_{\Gamma_{2}} \boldsymbol{T} \cdot \widetilde{\boldsymbol{\delta}} d S-\int_{\partial B} \boldsymbol{t} \cdot \widetilde{\boldsymbol{u}} d S- \\
\int_{B} \boldsymbol{f} \cdot \widetilde{\boldsymbol{u}} d V=0
\end{array}
$$

where $\boldsymbol{\sigma}$ is stress tensor, $\boldsymbol{T}$ the traction on the interface, $\boldsymbol{t}$ the applied traction on the 
external surface and $\boldsymbol{f}$ is the body force. $\tilde{\boldsymbol{\varepsilon}}$ is the virtual strain; $\widetilde{\boldsymbol{\delta}}$ and $\widetilde{\boldsymbol{u}}$ are virtual displacement jump and virtual displacement, respectively. $B_{P}$ and $B_{I}$ represent the volume of PTD and ITD, respectively; $\Gamma_{I}, \Gamma_{1}$ and $\Gamma_{2}$ stand for interfacial area, crack plane in PTD and crack plane in ITD, respectively. $B$ is the total volume containing $B_{P}$ and $B_{I}$, and $\partial B$ is the external surface area of $B$. The finite element procedure is adopted, where the displacement field, approximated by four-node isoparametric element (CPE4R in ABAQUS 6.10), is the nodal variable. A total of approximately 8000 elements is involved in the model. To capture crack growth, the refined mesh in the crack plane should be adopted. Previous studies reported that 2 5 cohesive elements in the cohesive zone are sufficient to characterize crack propagation in materials (Falk et al., 2001; Turon et al., 2007). Here, the relative length of cohesive element is $l_{e} / L=0.0067$ and approximately 100 cohesive elements are adopted in the cohesive zone, which satisfies the requirement of the minimum number of elements (Falk et al., 2001; Turon et al., 2007) and can effectively capture the crack growth in dentin. With this method, the stress and strain distributions in the microstructure of dentin can be obtained. To assess the toughness of dentin, we calculate the total energy $\left(W_{t}\right)$ of the two-layered structure up to failure, which is defined as the failure of any of the three phases (PTD, ITD and interface). The total energy, including the elastic strain energy of PTD and ITD and the damage dissipation energy of the interface and crack planes in PTD and ITD, can be calculated as

$$
W_{t}=\int_{0}^{t_{f}} \int_{B_{P}+B_{I}} \boldsymbol{\sigma}: \dot{\boldsymbol{\varepsilon}} d V d t+\int_{0}^{t_{f}} \int_{\Gamma_{I}+\Gamma_{1}+\Gamma_{2}} \frac{1}{2} \frac{\sigma}{\delta_{c}} \hat{\delta}^{2} \dot{D} d S d t
$$

where $t_{f}$ denotes the time corresponding to the failure of the two-layered structure and 
$\dot{\boldsymbol{\varepsilon}}$ is strain rate. This physical quantity is effective in characterizing the toughness of materials (Rim et al., 2011). Numerical simulations are performed to assess the influence of microstructure on the fracture of dentin. According to Buckingham's $\Pi$ theorem in dimensional analysis, it can be shown that the toughness, measured by the total energy, has the form

$$
\frac{W_{t}}{U_{0}}=\Pi\left(\frac{\sigma_{1}}{\sigma_{i}}, \frac{G_{1}}{G_{i}}, \frac{\delta_{c 1}}{\delta_{c i}}, \frac{\sigma_{i}}{\sigma_{2}}, \frac{G_{i}}{G_{2}}, \frac{\delta_{c i}}{\delta_{c 2}}, \frac{E_{1}}{E_{2}}, f_{v}, f_{1}, \frac{\delta_{c 2}}{L}\right)
$$

where $\sigma_{1}, \sigma_{i}$ and $\sigma_{2}$ are the strengths of PTD, interface and ITD, respectively; $G_{l}, G_{i}$ and $G_{2}$ are the fracture energies of PTD, interface and ITD, respectively. $\delta_{c l}, \delta_{c i}$ and $\delta_{c 2}$ are the critical separations of PTD, interface and ITD, respectively, and $E_{1}$ and $E_{2}$ are the elastic modulus of PTD and ITD, respectively. $f_{v}$ and $f_{l}$ are respectively volume fractions of dentin tubules and PTD, and $U_{0}$ is given by $U_{0}=\sigma_{2} L^{2}$. In all the numerical simulations, we set $L=3 \mu \mathrm{m}$, which is comparable to the geometry of dentin microstructure, and $\delta_{c 2}=0.039 \times 10^{-5} \mu \mathrm{m}$, which represents the brittle fracture properties. Based on these parameters, the normalized critical separation is $\delta_{c 2} / L=0.013 \times 10^{-5}$

\section{Results and discussion}

\subsection{Effect of mechanical properties of PTD on the fracture of dentin}

The variation of toughness $W_{t} / U_{0}$ with strength $\sigma_{l} / \sigma_{i}$ and critical separation $\delta_{c l} / \delta_{c i}$ is shown in Fig. 2(a). It is found that in the case of small critical separation $\left(\delta_{c l} / \delta_{c i}\right)$, the toughness of dentin initially increases with the increase of PTD strength $\left(\sigma_{l} / \sigma_{i}\right)$, then reaches a saturation value $W_{t} / U_{0}=3.176 \times 10^{-4}$. However, the effect of PTD strength on 
the toughness of dentin is complex in the case of large critical separation, $\delta_{c l} / \delta_{c i}=1$. The toughness initially decreases with increasing PTD strength, reaching a minimum value of $2.373 \times 10^{-4}$ when the normalized PTD strength reaches 0.4. As the PTD strength further increases, the toughness increases and reaches a saturation value which is identical to that in the case of small critical separation. This suggests that $\sigma_{1} / \sigma_{i}=1$ is a critical value, beyond which the toughness of dentin is not influenced by the PTD strength and critical separation. It can also be observed that when the PTD strength is smaller than that of the interface, the toughness of dentin increases with the increase in critical separation $\delta_{c l} / \delta_{c i}$. Fig. 2(b) shows the stress contour of the two-layered structure of dentin in the failure state. Based on preliminary numerical simulations, when cracking propagates to a length of $90 \%$ of the length of initial undamaged cohesive zone, the material loses load-carrying capacity and hence this is considered to fracture. Under this assumption, it can be found that the failure mechanism of dentin in these cases is fracture of PTD (Fig. 2b). Thus although the interfacial cracking occurs during the fracture of PTD, complete fracture of interface does not take place. It can be further concluded that the cracking length of the interface is influenced by the PTD strength $\left(\sigma_{l} / \sigma_{i}\right)$; with increasing $\sigma_{l} / \sigma_{i}$, the length of the interface crack increases, indicating that compared with the case of small strength, there are more areas participating in dissipating energy in the case of large strength, which contributes to the enhanced toughness. To explore the mechanisms responsible for the complex variation trend of toughness in the case of $\delta_{c l} / \delta_{c i}=1$, we calculated the total energy as a function of overall strain. As shown in Fig. 2 c, at the same 
overall strain, the total energies for the four cases $\left(\sigma_{1} / \sigma_{i}=0.05,0.2,0.4\right.$ and 0.7$)$ are identical, whereas the failure strain depends on the PTD strength (Fig. 2d). With increasing PTD strength $\left(\sigma_{l} / \sigma_{i}\right)$, the failure strain first decreases and then reaches the minimum value of 0.012 at the PTD strength $\left(\sigma_{1} / \sigma_{i}\right)$ of 0.4 . As the PTD strength further increases, the failure strain exhibits an increasing trend. Therefore, the underlying deformation mechanism is that the PTD strength has an influence on the failure strain of the microstructure of dentin, which leads to the complex variation of toughness. It should be noted that the failure mechanism predicted by numerical simulations (Section 2) is consistent with experimental observations (Eltit et al., 2013), suggesting that the numerical model can be used to characterize the failure mechanisms of dentin effectively.

The change of shape in tubules after fracture was also investigated. As shown in Fig. 2e, the circular cross sections of tubules evolve into ellipses with the long axes being parallel to the 2-axis. The length of the long axis of elliptic cross section is affected by PTD strength and its variation trend is similar to that of toughness. The underlying mechanism contributing to such a variation is that the length of long axis is dominated by the failure strain; the increase in failure strain gives rise to the increase in the length of long axis. Fig. 3 shows the effect of fracture energy of PTD $\left(G_{l} / G_{i}\right)$ on the toughness of dentin. Interestingly, the toughness of dentin is independent of the fracture energy of the PTD. 


\subsection{Effect of mechanical properties of interface on the fracture of dentin}

The effects of interfacial strength and critical separation of interface on the toughness of dentin are shown in Fig. 4(a). The toughness increases with the increase in interface strength $\left(\sigma_{i} / \sigma_{2}\right)$, and the increasing rate, measured by the slope of the curves, is higher in the case of large interface strength compared with that in the case of small interface strength. This implies that a strong interface could benefit the toughness of dentin. It can be seen from Fig. 4(a) that as the critical separation of interface $\left(\delta_{c i} / \delta_{c 2}\right)$ increases, the toughness of dentin also increases. Examining the stress contour of dentin in the failure state, it is found that in the case of $\delta_{c i} / \delta_{c 2}=0.01$, the typical failure mechanism of dentin corresponding to the low interface strength $\left(\sigma_{i} / \sigma_{2}=0.2,0.6\right)$ is interfacial debonding, while it is PTD fracture for high interface strength $\left(\sigma_{i} / \sigma_{2} \geq 1\right)$, as shown in Fig. $4 \mathrm{~b}$. In the case of large critical separation of interface $\left(\delta_{c i} / \delta_{c 2} \geq 0.4\right)$, the failure mechanism of dentin is PTD fracture, no matter how small the interface strength is (Fig. 4c). Based on these results, it can be concluded that the interface strength and critical separation of interface are the two key factors governing the failure mechanism of dentin. A low interface strength and small critical separation of interface can give rise to interfacial debonding, while an increase in these two key parameters leads to a transition from interfacial debonding to PTD fracture.

In the bending experiment of dentin conducted by Eltit et al (2013), the microcracks nucleating in PTD propagate across the interface through the ITD and finally merge (in the ITD) with microcracks emanating from an adjacent PTD, 
whereas some microcracks seem to be arrested by the interface. Why does dentin display these two types of damage behavior is still unknown. The present study addresses this issue. Due to the heterogeneity of the microstructure of dentin, the two-layered structure with the interface having low strength and small critical separation leads to the interfacial debonding before the fracture of PTD, thereby suppressing the growth of microcracks nucleating in PTD and playing a role in crack arrest. However, in the case of a structure with the interface possessing high strength and large critical separation, the interface is so strong that interfacial debonding cannot occur easily. As a consequence, the microcracks in PTD can extend towards ITD and coalesce with other microcracks. The results can also provide insights for understanding age related degradation in mechanical properties of dentin. The studies by Jameson et al (1993) and Arola and Reprogel (2005) also reveal the two typical damage behaviors of dentin caused by aging. To explain why the old dentin cannot display interfacial debonding between PTD and ITD, it is proposed that the interface becomes stronger with aging (Arola and Reprogel, 2005; Bajaj et al., 2006). Although the present study is not focused on the effect of aging, it could still provide a mechanistic understanding. From a mechanics point of view, the high strength of interface can indeed resist interfacial debonding, nevertheless the small critical separation is also required. Fig.5 shows the effect of fracture energy of interface $\left(G_{i} / G_{2}\right)$ on the toughness of dentin. Like the effect of PTD fracture energy, the fracture energy of interface contributes little to dentin toughness.

\subsection{Effect of elastic modulus mismatch on the fracture of dentin}


The effect of elastic modulus mismatch $\left(E_{1} / E_{2}\right)$ between PTD and ITD on the toughness of dentin is also of interest. As shown in Fig. 6a, for $E_{1} / E_{2} \leq 2.5$, the toughness first decreases with increasing elastic modulus mismatch $E_{1} / E_{2}$ and then increases when elastic modulus mismatch $E_{1} / E_{2}$ is larger than 2 . For $E_{1} / E_{2}>2.5$, a decreasing trend in toughness with elastic modulus mismatch $E_{1} / E_{2}$ is observed. Fig. 6b shows stress contour of dentin microstructure in the failure state. The failure mechanism for the three considered cases is PTD fracture, suggesting that the elastic modulus mismatch cannot influence the failure mechanism of dentin microstructure. The area of the interface undergoing cracking in the case of $E_{1} / E_{2}=1$ is larger than that in the two other cases, which contributes to the large toughness. To identify the underlying mechanisms responsible for the local minimum and maximum toughness of dentin, the variation of total energy with overall strain was investigated. As shown in Fig. 6c, at the same overall strain, the total energies in the seven cases studied in this study $\left(E_{1} / E_{2}=1,1.5,2,2.2,2.5,3\right.$ and 4$)$ are approximately equal, whereas the failure strain shows a variation trend similar to toughness (Fig. 6d), indicating that the local minimum and maximum failure strain leads to the corresponding minimum and maximum toughness. Based on the results shown in Fig. 6a, it can be deduced that the elastic modulus mismatch of dentin should be in the range of 1 to 2.5 to amplify the toughness.

Using nanoindentation, Ziskind et al (2011) reported that the elastic moduli for PTD and ITD are 29.3 $\pm 6.7 \mathrm{GPa}$, and 17.4 $\pm 3.5 \mathrm{GPa}$, respectively. The elastic modulus mismatch between PTD and ITD is therefore in the range $1.08 \sim 2.59$. It can be seen 
that the numerical simulations show good agreement with experiment, substantiating that the numerical model developed in this study can capture the major mechanical behavior of dentin, and is a useful tool to investigate the deformation mechanisms of dentin. Furthermore, this study sheds new light on the mechanical robustness of dentin. After long-term natural selection, dentin has evolved into a unique two-layered structure. An interesting strategy of enhancing toughness provided by such a two-layered structure is to confine the elastic modulus mismatch to the range of 1.08 to 2.59. This also could explain why dentin exhibits the elastic modulus ratio of 1.08 $\sim 2.59$.

\subsection{Effect of volume fraction of dentin tubules on the fracture of dentin}

The effect of the volume fraction of dentin tubules $\left(f_{v}\right)$ on the toughness of dentin is shown in Fig. 7a. The normalized toughness for $f_{v}=0.022$ is $5.1375 \times 10^{-6}$, while the toughness for $f_{v}=0.196$ reaches up to $2.7498 \times 10^{-5}$, implying that large volume fraction of dentin tubules $f_{v}$ could promote toughness. Despite the distinct toughness, the failure mechanism of the two-layered structure in the three cases is PTD fracture, suggesting that increasing the volume fraction of dentin tubules $f_{v}$ cannot lead to the transition in failure mechanism (Fig. 7b). To explore the underlying mechanism contributing to the exceptional toughness caused by the large volume fraction of dentin tubules $f_{v}$, we calculated the elastic strain energy and damage energy dissipation. As shown in Fig. 7c, for the same overall strain, the dissipated damage energy in the case of $f_{v}=0.022$ is larger than that in the other two cases, whereas with 
an increase in volume fraction of dentin tubules $f_{v}$, the failure strain of the two-layered structure increases, which leads to a large toughness. For the energy storage capacity, the three cases display the same elastic energy at the same strain, whereas due to the large failure strain, the stored elastic energy up to failure in the case of $f_{v}=0.196$ is larger than that in the other two cases (Fig. 7d). Hence, the underlying mechanism is that the large volume fraction of dentin tubules gives rise to large failure strain of the two-layered structure of dentin, which leads to the enhanced toughness.

\subsection{Effect of volume fraction of PTD on the fracture of dentin}

Fig. 8a shows the effect of volume fraction of PTD $\left(f_{1}\right)$ on the toughness of dentin. The normalized toughness for $f_{1}=0.109$ is $2.626 \times 10^{-5}$, while it is reduced to $5.358 \times 10^{-6}$ when the volume fraction of $\operatorname{PTD} f_{1}$ increases to 0.458 , suggesting that low volume fraction of PTD could promote toughness. The stress contour of the two-layered structure of dentin in failure state is shown in Fig. 8b. All three cases display PTD fracture, implying that the failure mechanism of dentin is not dependent on $f_{1}$. In spite of the same failure mechanism, the damage of the interface is affected by the volume fraction of PTD $f_{1}$. The area of the interface undergoing cracking for low volume fraction of $\operatorname{PTD} f_{l}$ is larger than that for high $f_{l}$, indicating that compared to the case of high volume fraction of $\operatorname{PTD} f_{l}$, there are more areas participating in energy dissipation in the case of low $f_{1}$, which contributes to the enhanced toughness. Additionally, when subjected to tensile loading, the ligament between the two adjacent tubules contracts in the case of $f_{1}=0.458$ (Fig. 8b), leading to the 
compression deformation of the interface, which suppresses the nucleation of cracks there. We also calculated the damage dissipation energy and elastic strain energy. As shown in Fig. 8c, the case of $f_{l}=0.109$ leads to larger failure strain than the other two cases, which gives rise to large energy dissipation. Such large failure strain can improve the energy storage capacity, as seen in Fig. 8d. In summary, decreasing the volume fraction of PTD $f_{l}$ could lead to a large failure strain and to an increase in damaged areas of interface, both of which contribute to the amplified toughness.

Wang (2005) experimentally reported that root dentin shows greater fracture resistance than coronal dentin. However, the underlying mechanisms responsible for this toughening in root dentin are not fully understood. The study by Ivancik and Arola (2013) revealed that the fracture toughness of outer coronal dentin is higher than that of middle coronal dentin, reflecting the graded fracture resistance of coronal dentin. Obviously, the toughening mechanisms in root dentin and outer coronal dentin are closely associated with the microstructure. It is known that the thickness of PTD in coronal dentin is larger than that in root dentin and that outer coronal dentin has a thinner PTD compared with the middle coronal dentin (Schilke et al., 2000; Chu et al., 2010; Ivancik and Arola, 2013). According to the present study, the thin PTD layer in root dentin and outer coronal dentin enables large failure strain and more areas in interface participating in energy dissipation; these mechanisms synergistically contribute to the enhanced toughness. The two mechanisms caused by the thin PTD layer can also provide a deeper insight into the deterioration in mechanical properties of old dentin. In old dentin, deposition of mineral within dentin tubules is found, 
leading to a thicker PTD and a lower volume fraction of tubules (Bajaj et al., 2006; Nazari et al., 2009). Based on this study, the two changes can lead to the reduction in toughness of dentin, which offers an additional understanding of the mechanisms responsible for the poor mechanical performance of old dentin.

\section{Conclusions}

This study explored the effects of a two-layered microstructure on the fracture of dentin based on a numerical model, which incorporates the major damage mechanisms of PTD, ITD and the interface. Computational simulations show that there exists a critical value of the PTD strength, i.e. $\sigma_{l} / \sigma_{i}=1$, beyond which the toughness of dentin is independent of PTD strength and of the critical separation of PTD. When the PTD strength is lower than this critical value, the toughness of dentin increases with the increase in critical separation of PTD. The fracture energies of PTD and interface have a negligible effect on the toughness of dentin. For the effects of mechanical properties of interface, it is found that increases in interface strength and in critical separation of interface could lead to an increase in dentin toughness. The numerical simulations predict two major failure mechanisms of the microstructure of dentin, i.e. PTD fracture and interfacial debonding, which show good agreement with experimental observations. Furthermore, this study reveals that the key factors dominating the failure mechanisms of dentin are interfacial strength and the critical separation of the interface. The low interface strength and small critical separation of interface could give rise to interfacial debonding, while with an increase in these two 
physical quantities, the failure mechanisms of dentin undergo a transition from interfacial debonding to PTD fracture. This study also predicts a characteristic range for the elastic modulus mismatch between PTD and ITD, within which dentin toughness can be enhanced. The predicted characteristic range is consistent with the experimentally determined elastic modulus mismatch, which further substantiates the effectiveness of the numerical model. In addition, it is revealed that increasing the volume fraction of dentin tubules and decreasing the volume fraction of PTD could also increase the toughness of dentin, which provides an additional explanation for the region-dependent toughening in dentin and for the deterioration in mechanical performance of dentin caused by aging.

\section{Acknowledgement}

This research was made possible in part by the generosity of the Harold Perlman family. B.A. acknowledges the VATAT fellowship from Israel and the National Science Foundation of China \#11402141. H.D.W. is the recipient of the Livio Norzi Professorial Chair at the Weizmann Institute.

\section{References}

An B, Zhao X, Arola D, Zhang D, 2014. Fracture analysis for biological materials with an expanded cohesive zone model. J Biomech 47, 2244-2248.

An B, Zhang D, 2015. Bioinspired toughening mechanism: lesson from dentin. Bioinspir Biomim 10, 046010. 
Arola D, Reid J, Cox ME, Bajaj D, Sundaram N, Romberg E, 2007. Transition behavior in fatigue of human dentin: Structure and anisotropy. Biomaterials 28, $3867-3875$.

Arola D, Reprogel RK, 2005. Effects of aging on the mechanical behavior of human dentin. Biomaterials 26, 4051-4061.

Bajaj D, Sundaram N, Nazari A, Arola D, 2006. Age, dehydration and fatigue crack growth in dentin. Biomaterials 27, 2507-2517.

Bar-On B, Wagner HD, 2012a. Enamel and dentin as multi-scale bio-composites. J Mech Behav Biomed Mater 12, 174-183.

Bar-On B, Wagner HD, 2012b. Elastic modulus of hard tissues. J Biomech 45, 672-678.

Bertassoni LE, Stankoska K, Swain MV, 2012. Insights into the structure and composition of the peritubular dentin organic matrix and the lamina limitans. Micron 43, 229-236.

Chu CY, Kuo TC, Chang SF, Shyu YC, Lin CP, 2010.Comparison of the microstructure of crown and root dentin by a scanning electron microscopic study. J Dent Sci 5, 14-20.

Deymier-Black AC, Almer JD, Stock SR, Haeffner DR, Dunand DC, 2010.Synchrotron X-ray diffraction study of load partitioning during elastic deformation of bovine dentin. Acta Biomater 6, 2172-2180.

Eltit F, Ebacher V, Wang R, 2013. Inelastic deformation and microcracking process in human dentin. J Struct Biol 183, 141-148. 
Falk ML, Needleman A, Rice JR, 2001. A critical evaluation of cohesive zone models of dynamic fracture. J Phys IV, Proc 543-550.

Gao H, Ji B, Jager IL, Arzt E, Fratzl P, 2003. Materials become insensitive to flaws at nanoscale: lessons from nature. Proc Natl Acad Sci 100(10), 5597-5600.

Han CF, Wu BH, Chung CJ, Chung SF, Li WL, Lin JF, 2012. Stress - strain analysis for evaluating the effect of the orientation of dentin tubules on their mechanical properties and deformation behavior. J Mech Behav Biomed Mater 12, 1-8.

Ivancik J, Neerchal NK, Romberg E, Arola D, 2011. The reduction in fatigue crack growth resistance of dentin with depth. J Dent Res 90, 1031-1036.

Ivancik J, Majd H, Bajaj D, Romberg E, Arola D, 2012. Contributions of aging to the fatigue crack growth resistance of human dentin. Acta Biomater 8, 2737-2746.

Ivancik J, Arola D, 2013. The importance of microstructural variations on the fracture toughness of human dentin. Biomaterials 34, 864-874.

Iwamoto N, Ruse ND, 2003. Fracture toughness of human dentin. J Biomed Mater Res A 66(3), 507-512.

Jameson MW, Hood JA, Tidmarsh BG, 1993. The effects of dehydration and rehydration on some mechanical properties of human dentine. J Biomech 26, $1055-1065$.

Koester KJ, Ager III JW, Ritchie RO, 2008. The effect of aging on crack-growth resistance and toughening mechanisms in human dentin. Biomaterials 29, 1313-1328.

Mosler J, Scheider I, 2011. A thermodynamically and variationally consistent class of 
damage-type cohesive models. J Mech Phys Solids 59, 1647-1658.

Nalla RK, Kinney JH, Ritchie RO, 2003. Effect of orientation on the in vitro fracture toughness of dentin: the role of toughening mechanisms. Biomaterials 24, 3955-3968.

Nazari A, Bajaj D, Zhang D, Romberg E, Arola D, 2009. Aging and the reduction in fracture toughness of human dentin. J Mech Behav Biomed Mater 2, 550-559.

Rim JE, Zavattieri P, Juster A, Espinosa HD, 2011.Dimensional analysis and parametric studies for designing artificial nacre. J Mech Behav Biomed Mater 4, 190-211.

Ryou H, Romberg E, Pashley DH, Tay FR, Arola D, 2012.Nanoscopic dynamic mechanical properties of intertubular and peritubular dentin. J Mech Behav Biomed Mater 7, 3-16.

Schilke R, Lisson JA, Bauss O, Geurtsen W, 2000. Comparison of the number and diameter of dentinal tubules in human and bovine dentine by scanning electron microscopic investigation. Arch Oral Biol 45, 355-361.

Stock SR, Veis A, Telser A, Cai Z, 2011. Near tubule and intertubular bovine dentin mapped at the $250 \mathrm{~nm}$ level. J Struct Biol 176, 203-211.

Turon A, Davila CG, Camanho PP, Costa J, 2007. An engineering solution for mesh size effects in the simulation of delamination using cohesive zone models. Engng Fract Mech 74, $1665-1682$.

Tvergaard V, Hutchinson JW, 1992.The relation between crack growth resistance and fracture process parameters in elastic-plastic solids.J Mech Phys Solids 40, 
1377-1397.

Ural A, Vashishth D, 2006.Cohesive finite element modeling of age-related toughness loss inhuman cortical bone. J Biomech 39, 2974-2982.

Ural A, Vashishth D, 2007.Anisotropy of age-related toughness loss in human cortical bone: A finite element study. J Biomech 40, 1606-1614.

Wang R, 2005. Anisotropic fracture in bovine root and coronal dentin. Dent Mater 21, 429-436.

Xu XP, Needleman A, 1994. Numerical simulations of fast crack growth in brittle solids. J Mech Phys Solids 42, 1397 - 1434.

Xu Q, Lu Z, 2013. An elastic-plastic cohesive zone model for metal-ceramic interfaces at finite deformations. Int J Plast 41, 147 - 164.

Zaslansky P, Zabler S, Fratzl P, 2010. 3D variations in human crown dentin tubule orientation: A phase-contrast microtomography study. Dent Mater 26, e1-e10.

Ziskind D, Hasday M, Cohen SR, Wagner HD, 2011. Young's modulus of peritubular and intertubular human dentin by nano-indentation tests. J Struct Biol 174, 23-30. 


\section{Figure captions}

Figure 1.Model of microstructure of dentin. (a) Bilinear cohesive zone model. (b) Schematics of the microstructure of dentin and the unit cell model in numerical simulations.

Figure 2. Effects of PTD strength and the critical separation of PTD on the fracture of dentin. The materials parameters used in numerical simulations are $G_{l} / G_{i}=0.05, \sigma_{i} / \sigma_{2}$ $=0.4, G_{i} / G_{2}=1, \delta_{c i} / \delta_{c 2}=0.4, E_{1} / E_{2}=2, f_{v}=0.087$ and $f_{1}=0.262$. (a) Toughness of the two-layered structure of dentin. (b) von Mises stress contours of the damaged two-layered structure of dentin corresponding to $\delta_{c l} / \delta_{c i}=0.005$. (c) Variation of total energy with overall strain in the case of $\delta_{c l} / \delta_{c i}=1$. (d) Failure strain as a function of PTD strength in the case of $\delta_{c 1} / \delta_{c i}=1$.(e) The shape of dentin tubule after fracture occurs. xy represents the local coordinate system with the origin being the center of the circular cross section of dentin tubule. The $\mathrm{x}$-axis is parallel to the 1 -axis (shown in Fig. 1b) and the y-axis is parallel to the 2-axis. Note that the failure mechanisms in the cases of $\delta_{c l} / \delta_{c i}=0.1$ and $\delta_{c l} / \delta_{c i}=1$ are identical to that in the case of $\delta_{c l} / \delta_{c i}=$ 0.005 .

Figure 3. Effect of fracture energy of PTD $\left(G_{l} / G_{i}\right)$ with $\sigma_{l} / \sigma_{i}=0.4, \sigma_{i} / \sigma_{2}=0.4, G_{i} / G_{2}=$ $1, \delta_{c i} / \delta_{c 2}=0.4, E_{1} / E_{2}=2, f_{v}=0.087$ and $f_{1}=0.262$ on the toughness of dentin. 
Figure 4. Effects of interfacial strength and critical separation of interface on the fracture of dentin. The materials parameters used in numerical simulations are $\sigma_{l} / \sigma_{i}$ $=0.05, G_{1} / G_{i}=0.05, \delta_{c l} / \delta_{c i}=0.005, G_{i} / G_{2}=1, E_{1} / E_{2}=2, f_{v}=0.087$ and $f_{1}=0.262$. (a) Toughness of the two-layered structure of dentin. (b) von Mises stress contour of the damaged two-layered structure of dentin corresponding to $\delta_{c i} / \delta_{c 2}=0.01$. (c) von Mises stress contour of the damaged two-layered structure of dentin corresponding to $\delta_{c i} / \delta_{c 2}$ $=0.4$.

Figure 5. Effect of fracture energy of interface $\left(G_{i} / G_{2}\right)$ with $\sigma_{1} / \sigma_{i}=0.05, G_{1} / G_{i}=0.05$, $\delta_{c l} / \delta_{c i}=0.005, \sigma_{i} / \sigma_{2}=0.2, E_{1} / E_{2}=2, f_{v}=0.087$ and $f_{1}=0.262$ on the toughness of dentin.

Figure 6. Effect of elastic modulus mismatch $\left(E_{1} / E_{2}\right)$ on the fracture of dentin. The materials parameters used in numerical simulations are $\sigma_{l} / \sigma_{i}=0.05, G_{l} / G_{i}=0.05$, $\delta_{c 1} / \delta_{c i}=0.005, \sigma_{i} / \sigma_{2}=4, G_{i} / G_{2}=1, \delta_{c i} / \delta_{c 2}=0.4, f_{v}=0.087$ and $f_{1}=0.262$. (a) Toughness of the two-layered structure of dentin. (b) von Mises stress contour of the damaged two-layered structure of dentin. (c) The variations of total energy with overall strain. (d) The variation of failure strain with elastic modulus mismatch.

Figure 7. Effect of the volume fraction of dentin tubules on the fracture of dentin. The material parameters used in numerical simulations are $\sigma_{l} / \sigma_{i}=0.05, G_{l} / G_{i}=0.05, \delta_{c l} / \delta_{c i}$ $=0.005, \sigma_{i} / \sigma_{2}=4, G_{i} / G_{2}=1, \delta_{c i} / \delta_{c 2}=0.4, E_{1} / E_{2}=2$ and $f_{1}=0.262$. (a) Toughness of 
the two-layered structure of dentin. (b) von Mises stress contour of the damaged two-layered structure of dentin. (c) The variations of damage dissipation energy with overall strain. (d) The variations of elastic strain energy with overall strain.

Figure 8. Effect of volume fraction of PTD on the fracture of dentin. The material parameters used in numerical simulations are $\sigma_{l} / \sigma_{i}=0.05, G_{l} / G_{i}=0.05, \delta_{c l} / \delta_{c i}=$ $0.005, \sigma_{i} / \sigma_{2}=4, G_{i} / G_{2}=1, \delta_{c i} / \delta_{c 2}=0.4, E_{1} / E_{2}=2$ and $f_{v}=0.087$. (a) Toughness of the two-layered structure of dentin. (b) von Mises stress contour of the damaged two-layered structure of dentin. (c) The variations of damage dissipation energy with overall strain. (d) The variations of elastic strain energy with overall strain. 


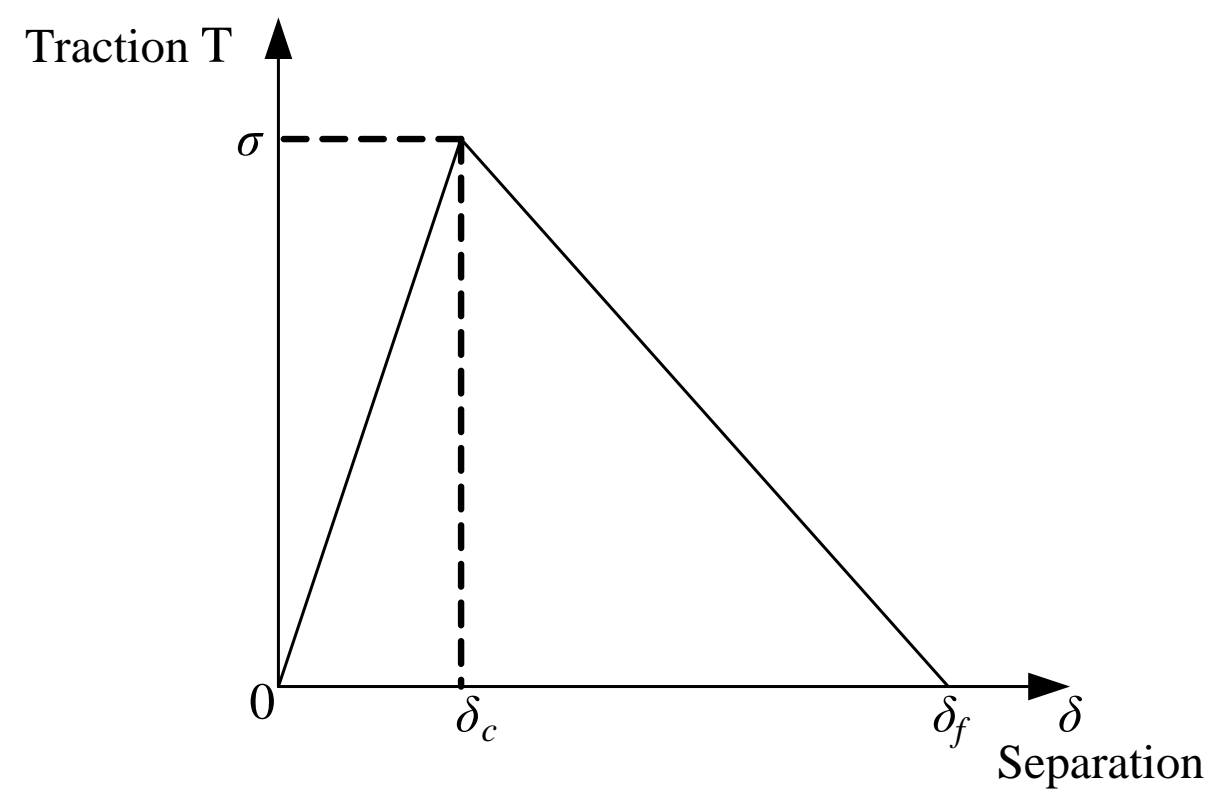

(a)

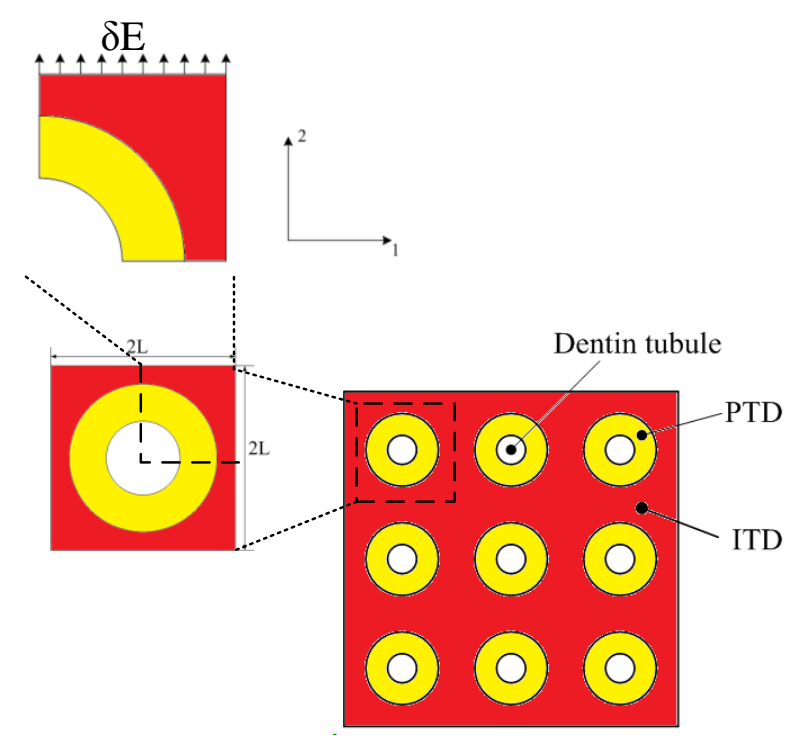

(b)

Figure 1. Model of microstructure of dentin. (a) Bilinear cohesive zone model. (b) Schematics of the microstructure of dentin and the unit cell model in numerical simulations. 


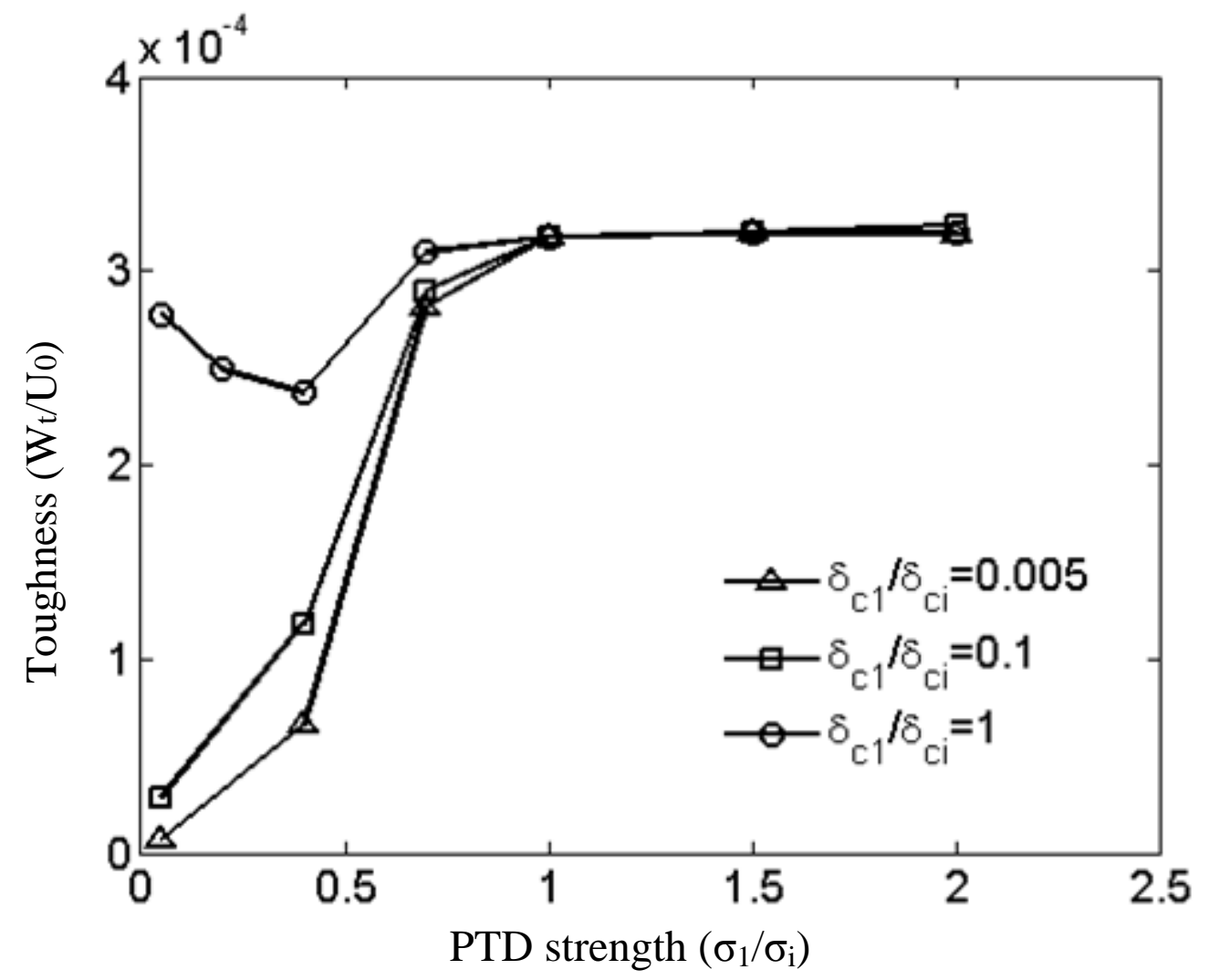

(a)

von Mises stress $\sigma_{\mathrm{g}} / \sigma_{2}$
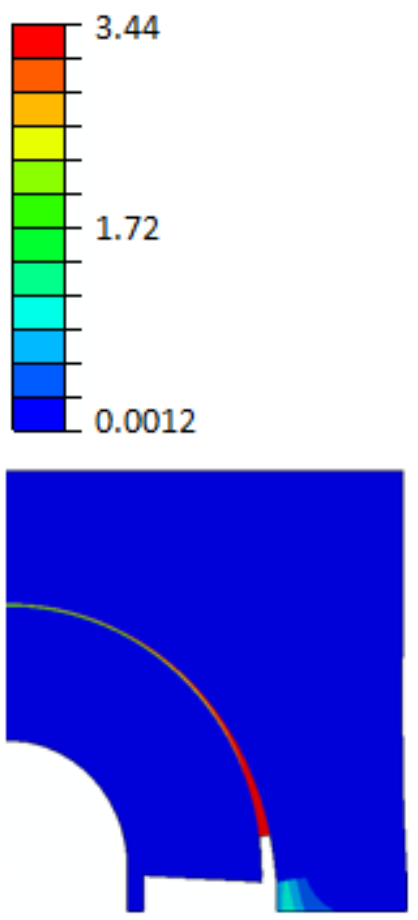

$\sigma_{l} / \sigma_{i}=0.05$
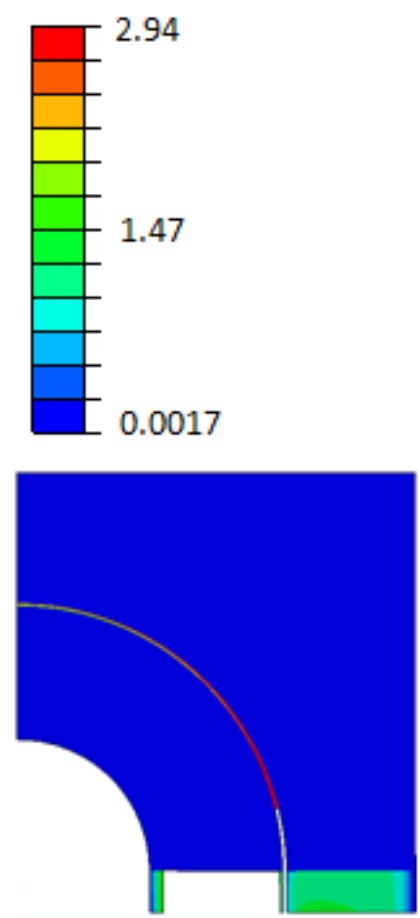

$\sigma_{l} / \sigma_{i}=0.4$
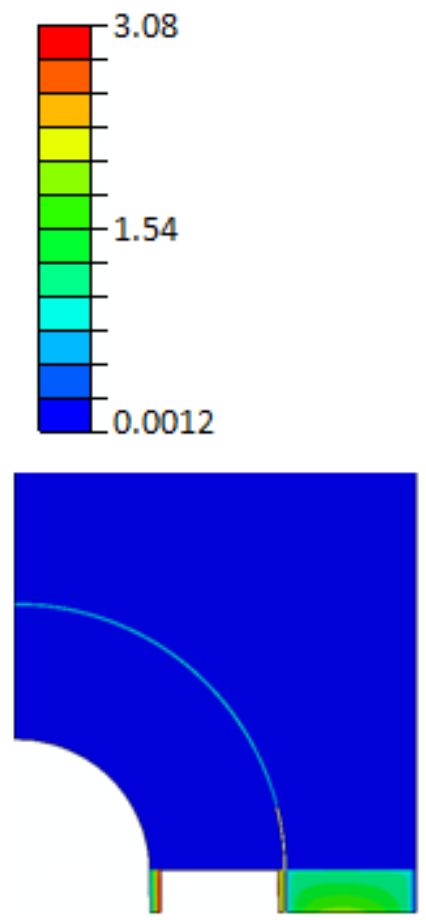

$\sigma_{l} / \sigma_{i}=0.7$ 
(b)

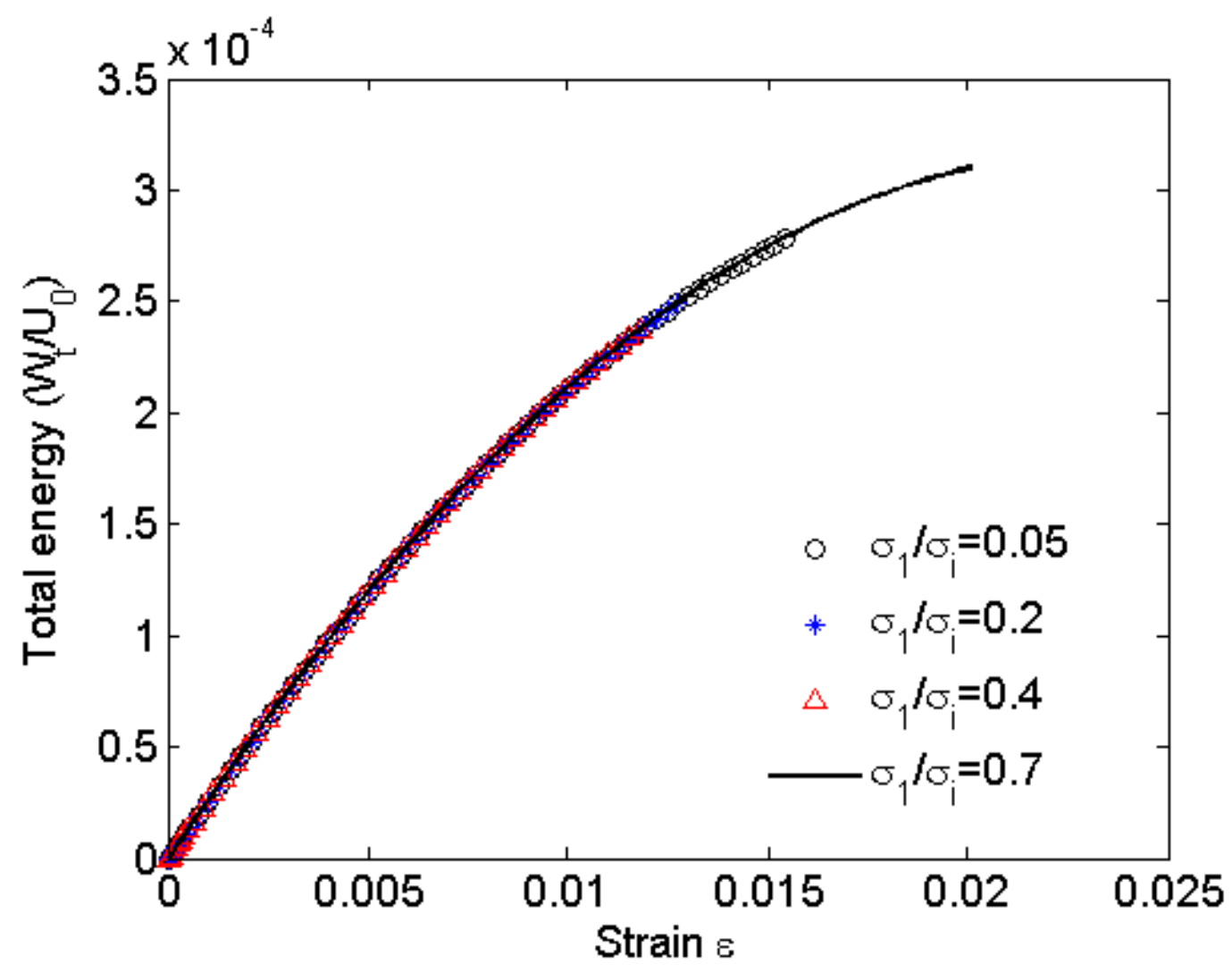

(c) 


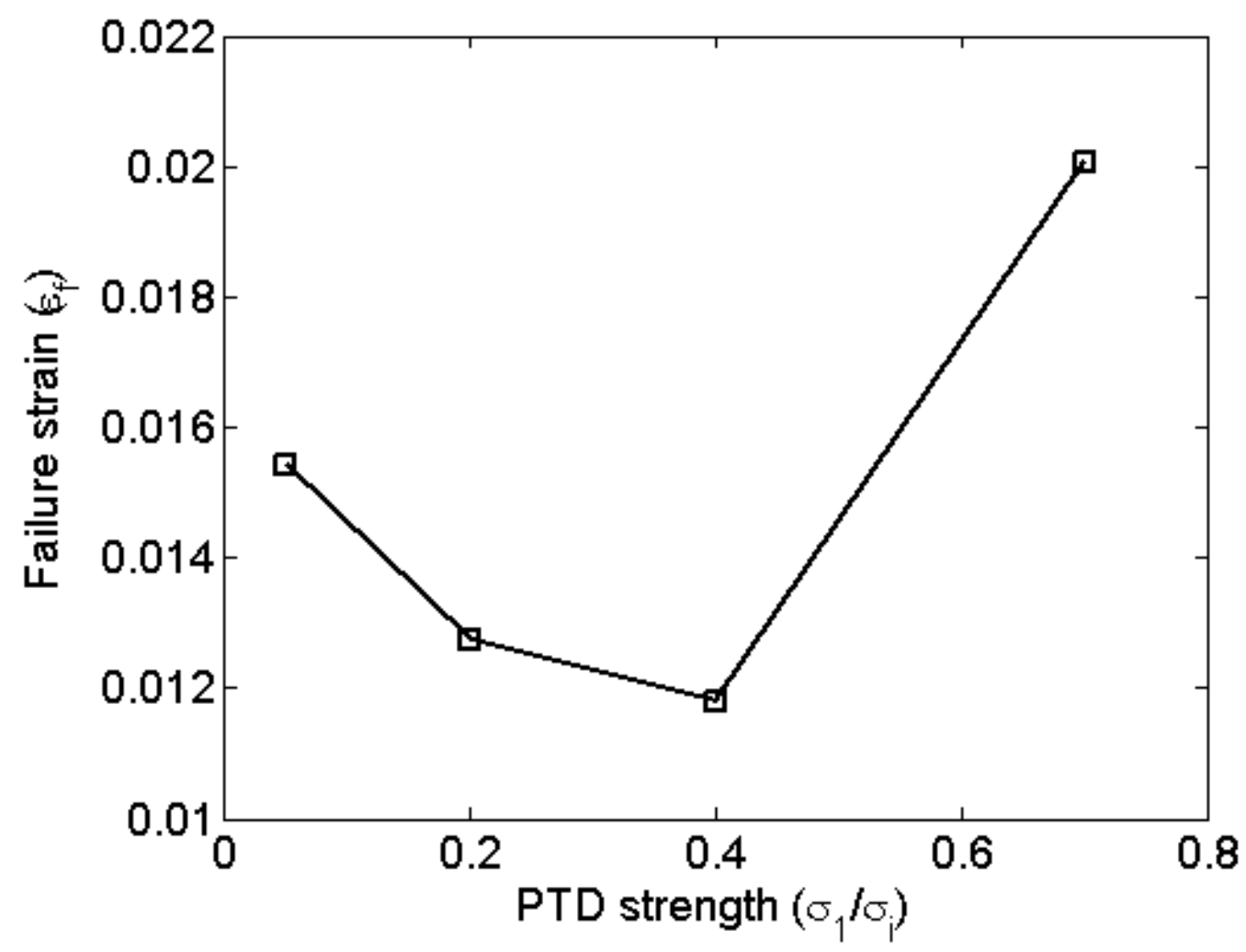

(d)

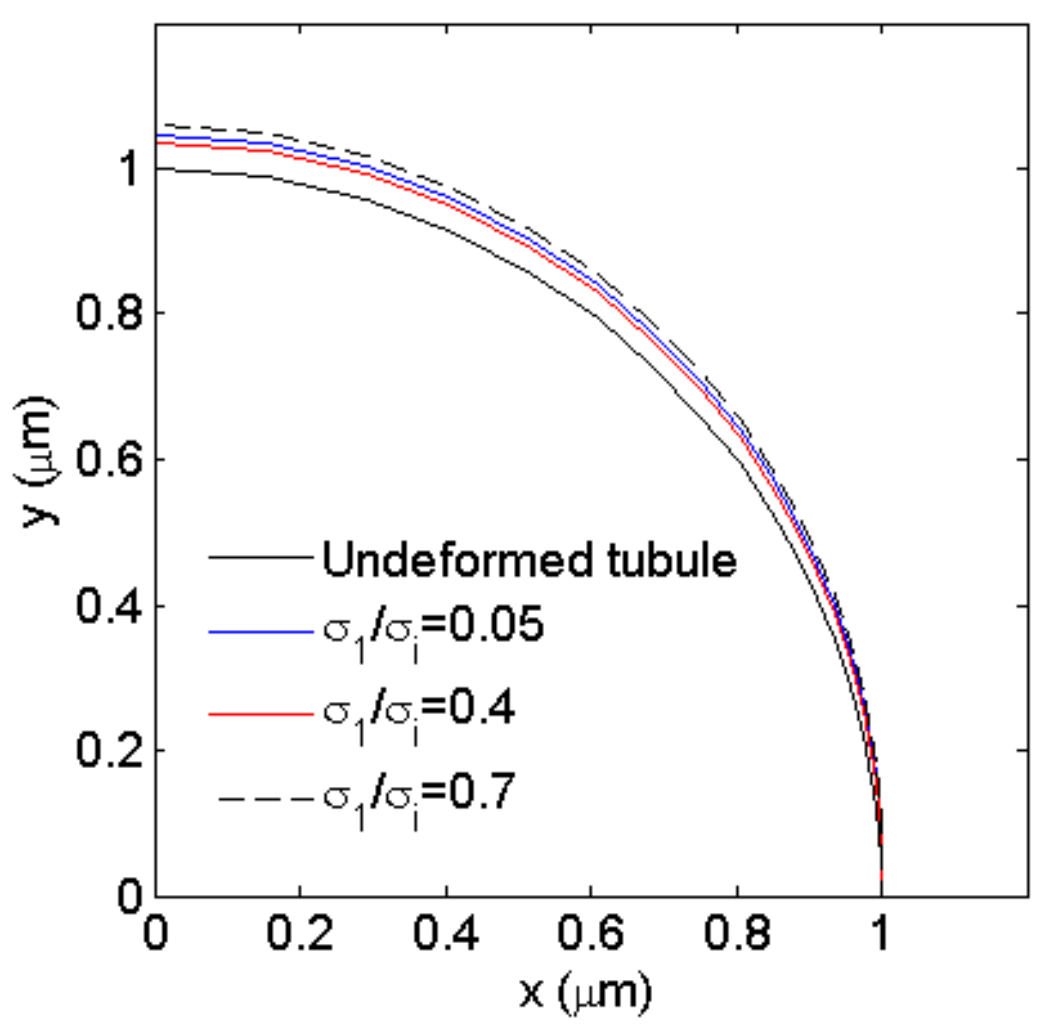

(e) 
Figure 2. Effects of PTD strength and the critical separation of PTD on the fracture of dentin. The materials parameters used in numerical simulations are $G_{l} / G_{i}=0.05, \sigma_{i} / \sigma_{2}$ $=0.4, G_{i} / G_{2}=1, \delta_{c i} / \delta_{c 2}=0.4, E_{1} / E_{2}=2, f_{v}=0.087$ and $f_{1}=0.262$. (a) Toughness of the two-layered structure of dentin. (b) von Mises stress contours of the damaged two-layered structure of dentin corresponding to $\delta_{c l} / \delta_{c i}=0.005$. (c) Variation of total energy with overall strain in the case of $\delta_{c l} / \delta_{c i}=1$. (d) Failure strain as a function of PTD strength in the case of $\delta_{c l} / \delta_{c i}=1$.(e) The shape of dentin tubule after fracture occurs. xy represents the local coordinate system with the origin being the center of the circular cross section of dentin tubule. The $\mathrm{x}$-axis is parallel to the 1 -axis (shown in Fig. 1b) and the y-axis is parallel to the 2-axis. Note that the failure mechanisms in the cases of $\delta_{c l} / \delta_{c i}=0.1$ and $\delta_{c l} / \delta_{c i}=1$ are identical to that in the case of $\delta_{c l} / \delta_{c i}=$ 0.005 . 


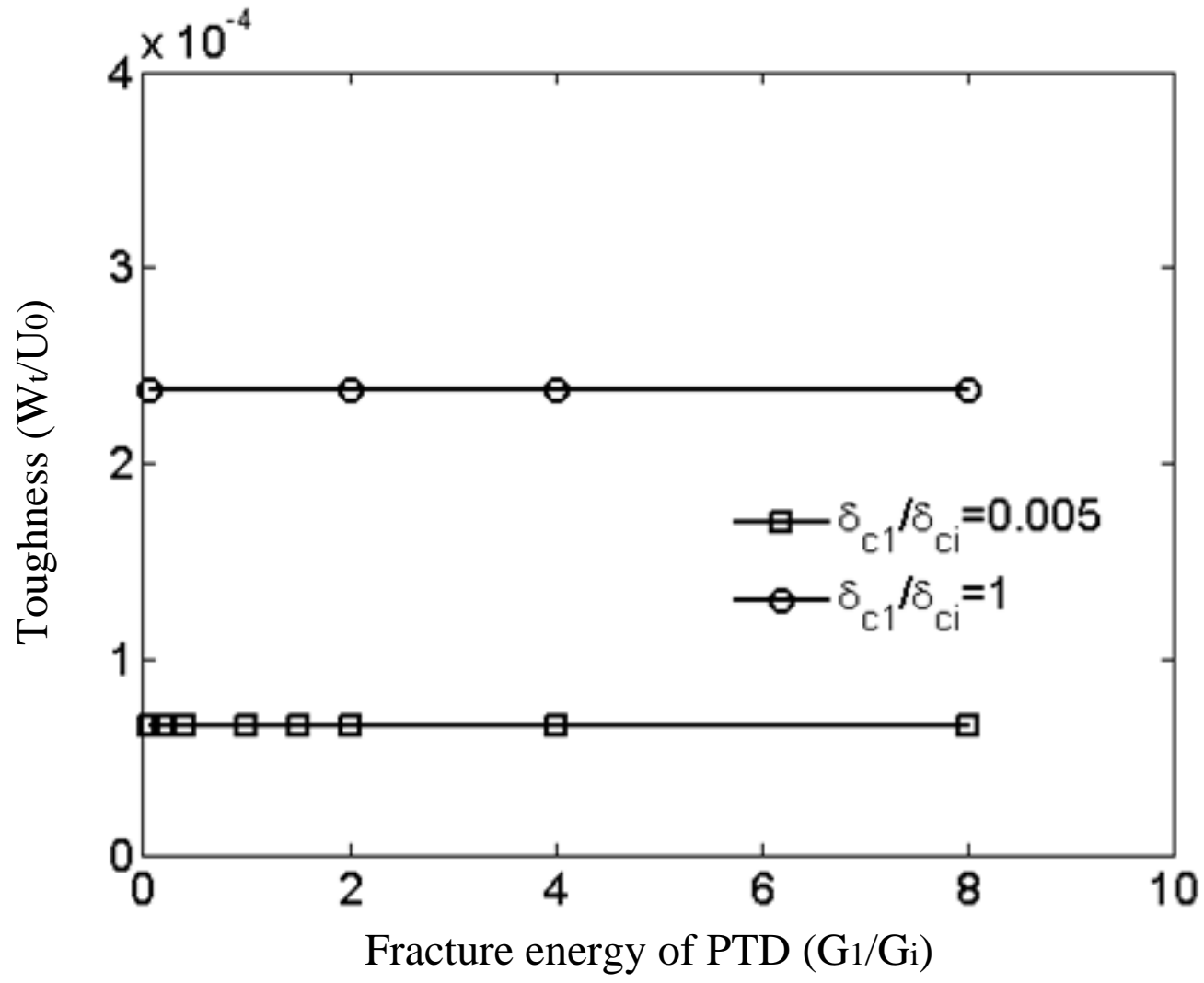

Figure 3. Effect of fracture energy of PTD $\left(G_{1} / G_{i}\right)$ with $\sigma_{1} / \sigma_{i}=0.4, \sigma_{i} / \sigma_{2}=0.4, G_{i} / G_{2}=$ $1, \delta_{c i} / \delta_{c 2}=0.4, E_{1} / E_{2}=2, f_{v}=0.087$ and $f_{1}=0.262$ on the toughness of dentin. 


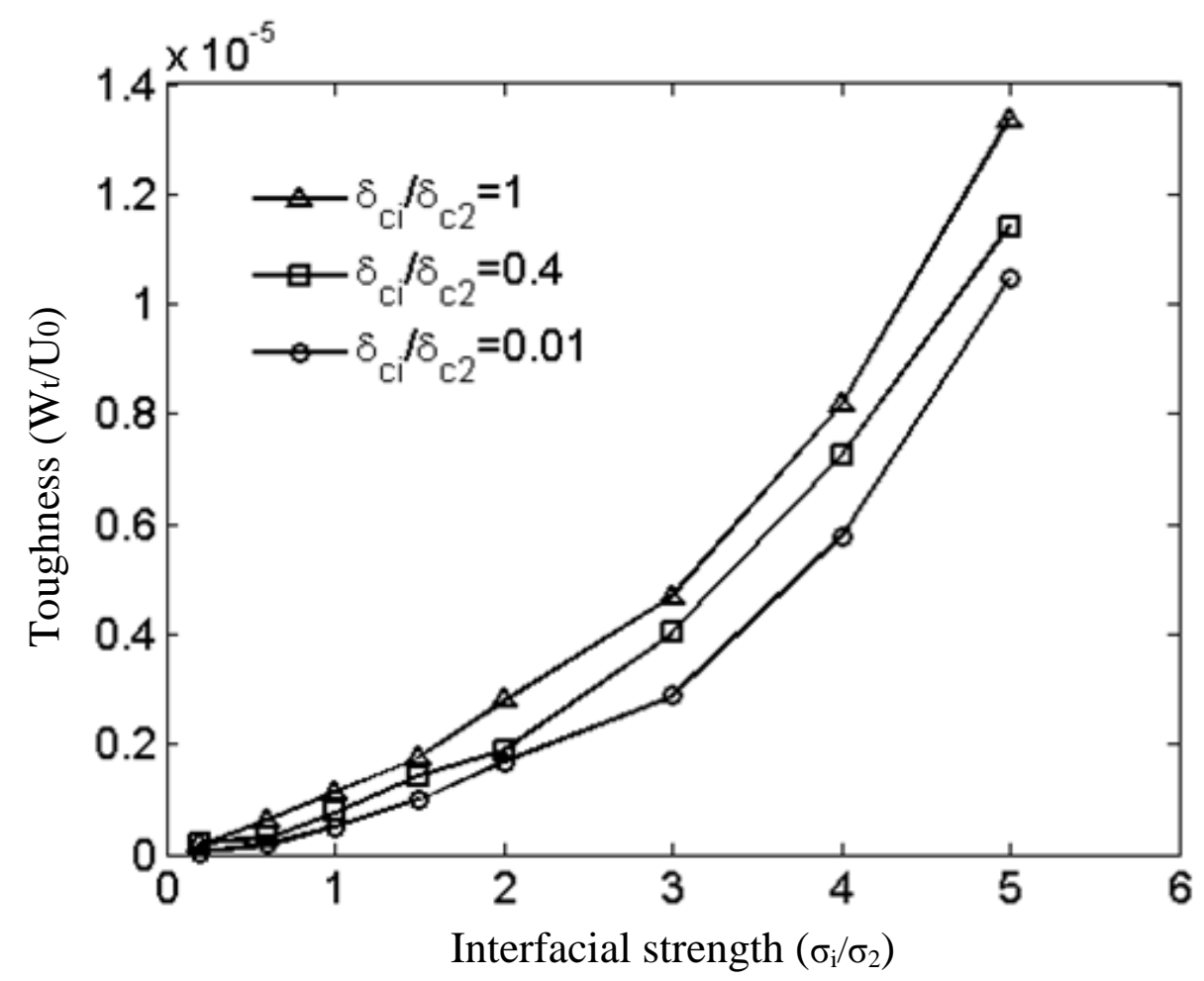

(a)

von Mises stress $\sigma_{e} / \sigma_{2}$
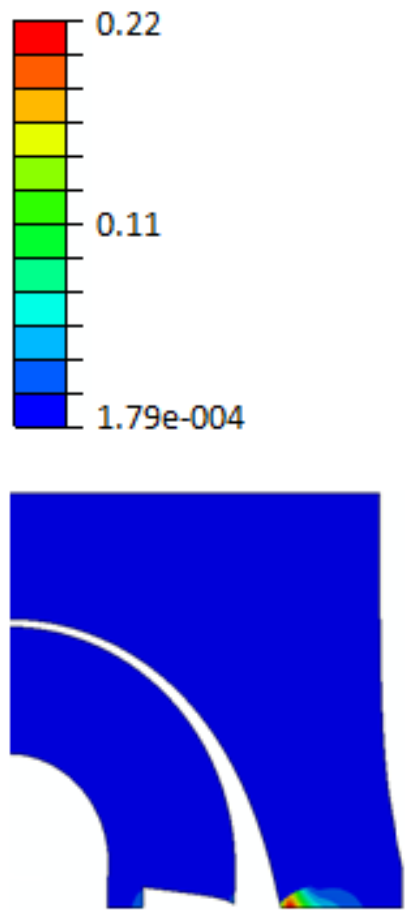

$\sigma_{i} / \sigma_{2}=0.2$
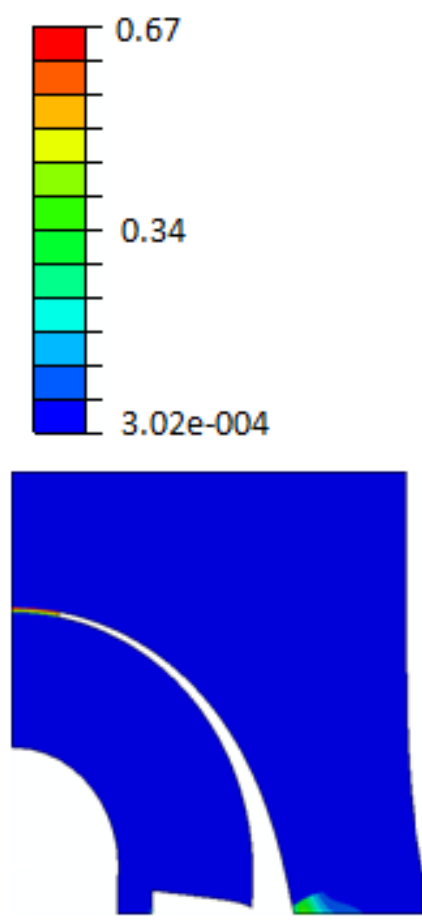

$\sigma_{i} / \sigma_{2}=0.6$
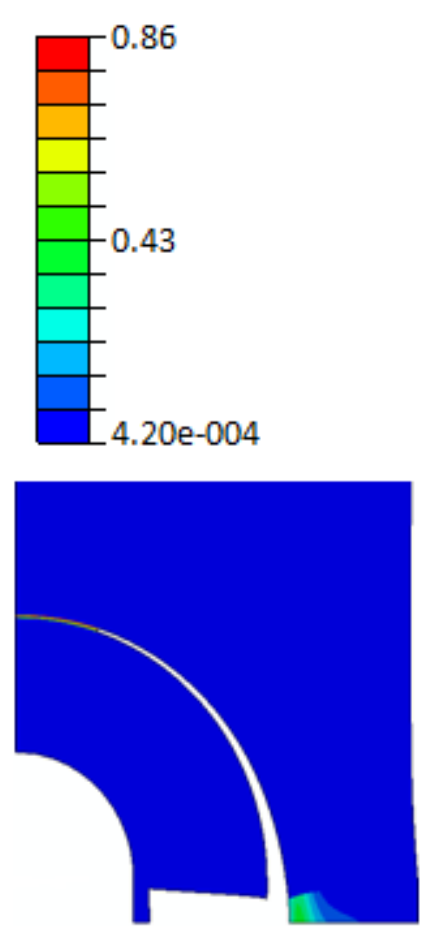

$\sigma_{i} / \sigma_{2}=1$ 
von Mises stress $\sigma_{\mathrm{e}} / \sigma_{2}$
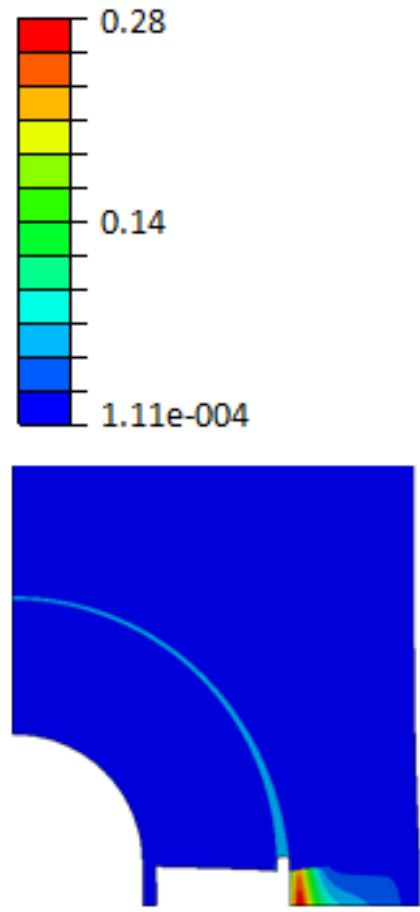

$\sigma_{i} / \sigma_{2}=0.2$
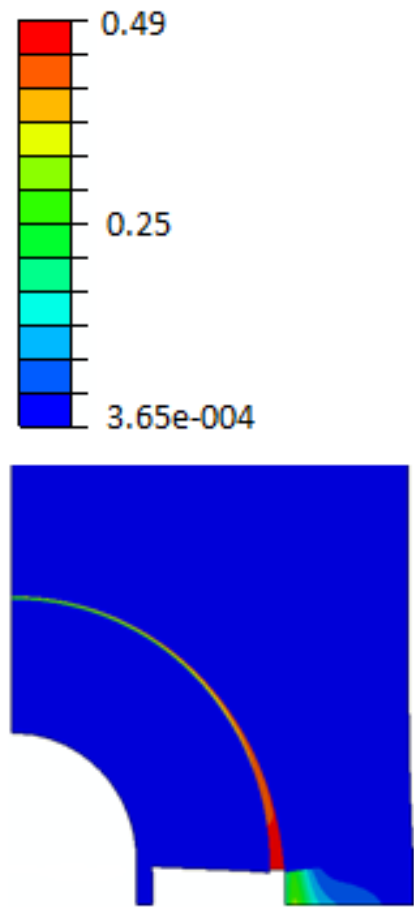

$\sigma_{i} / \sigma_{2}=0.6$
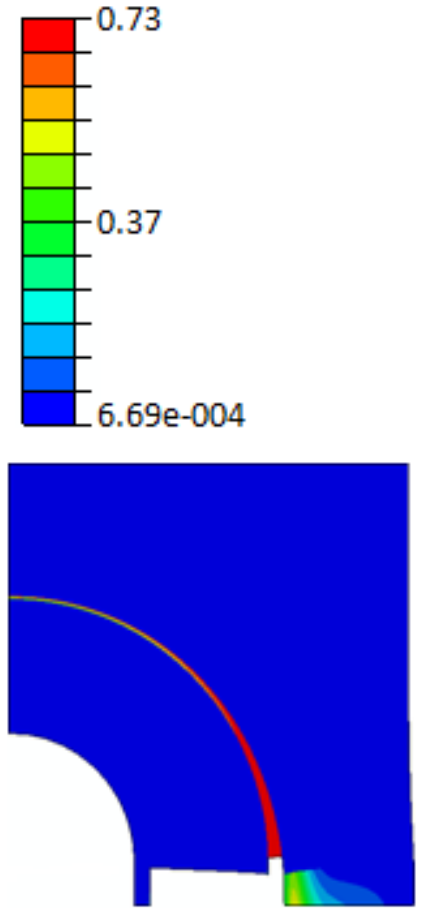

$\sigma_{i} / \sigma_{2}=1$

(c)

Figure 4. Effects of interfacial strength and critical separation of interface on the fracture of dentin. The materials parameters used in numerical simulations are $\sigma_{1} / \sigma_{i}$ $=0.05, G_{1} / G_{i}=0.05, \delta_{c l} / \delta_{c i}=0.005, G_{i} / G_{2}=1, E_{1} / E_{2}=2, f_{v}=0.087$ and $f_{1}=0.262$. (a) Toughness of the two-layered structure of dentin. (b) von Mises stress contour of the damaged two-layered structure of dentin corresponding to $\delta_{c i} / \delta_{c 2}=0.01$. (c) von Mises stress contour of the damaged two-layered structure of dentin corresponding to $\delta_{c i} / \delta_{c 2}$ $=0.4$. 


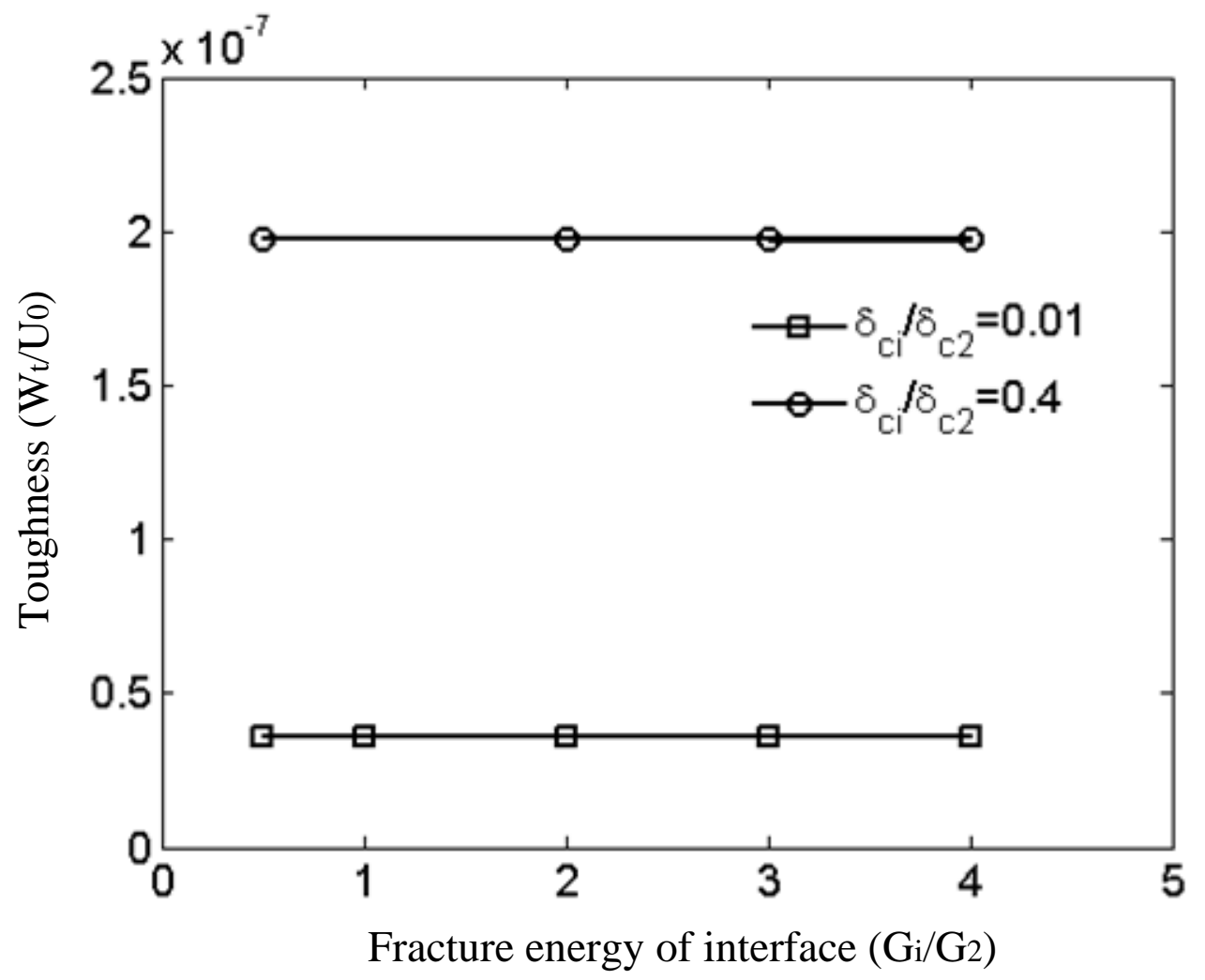

Figure 5. Effect of fracture energy of interface $\left(G_{i} / G_{2}\right)$ with $\sigma_{1} / \sigma_{i}=0.05, G_{l} / G_{i}=0.05$, $\delta_{c l} / \delta_{c i}=0.005, \sigma_{i} / \sigma_{2}=0.2, E_{1} / E_{2}=2, f_{v}=0.087$ and $f_{l}=0.262$ on the toughness of dentin. 


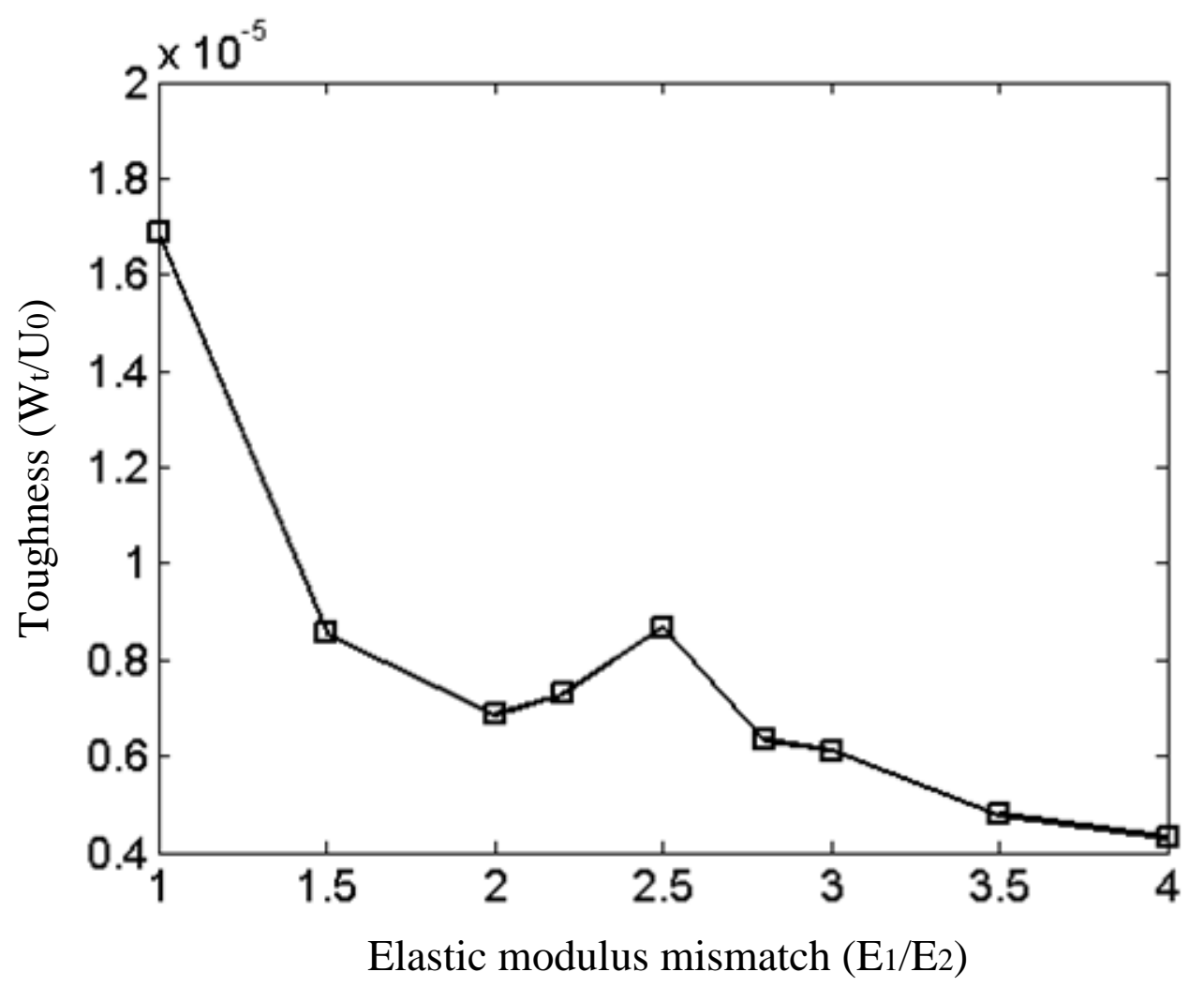

(a)

von Mises stress $\sigma_{2} / \sigma_{2}$
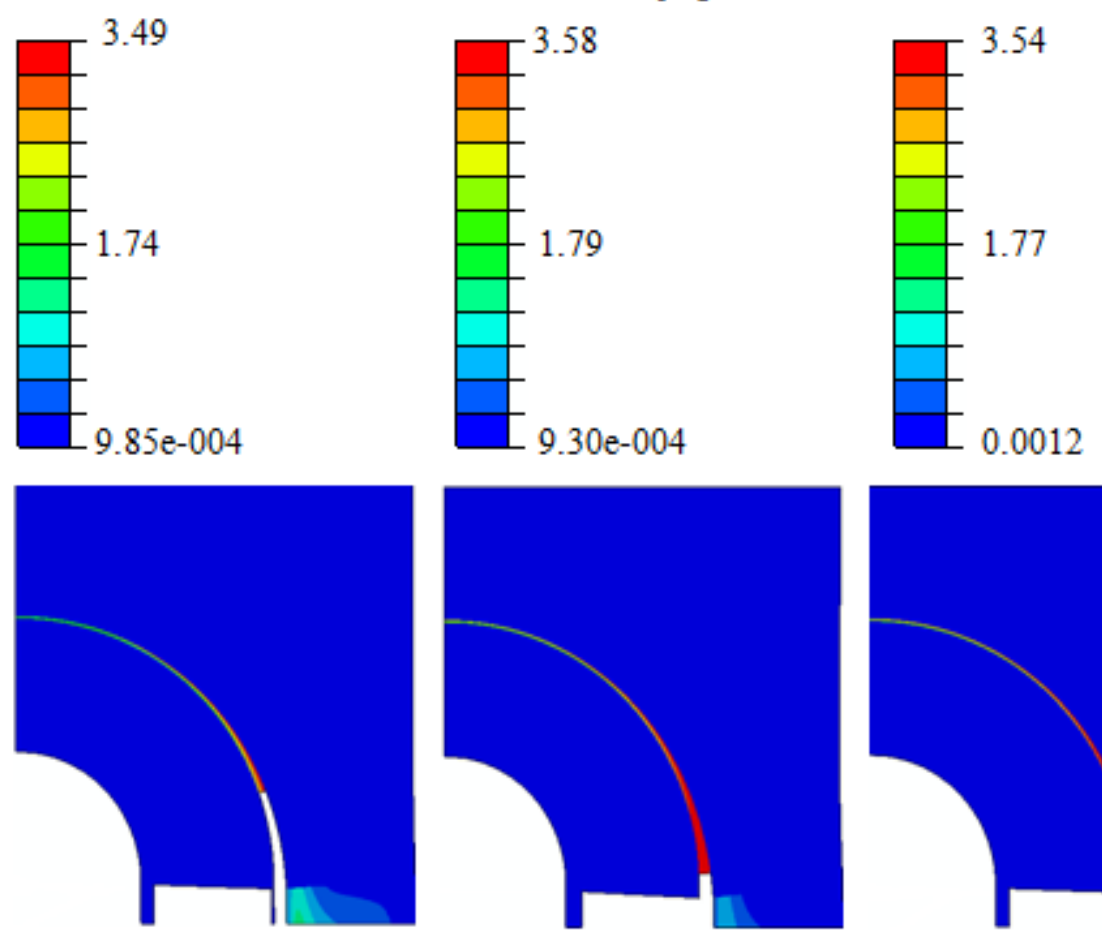

$E_{1} / E_{2}=1$
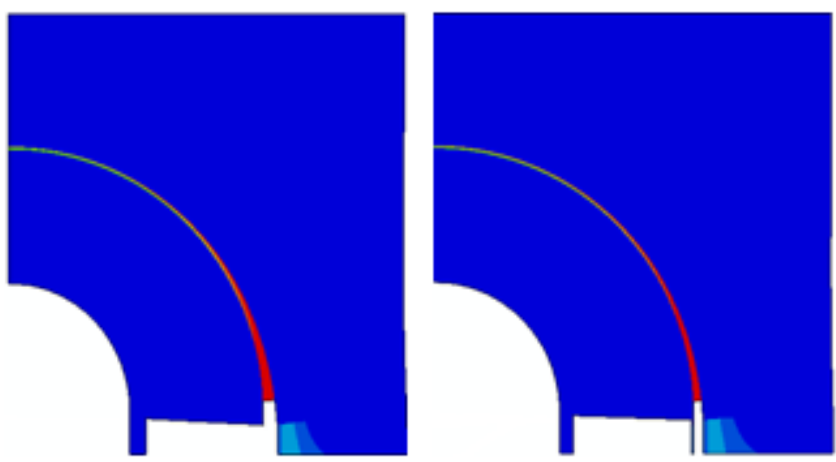

$E_{1} / E_{2}=2$

$E_{1} / E_{2}=2.5$ 
(b)

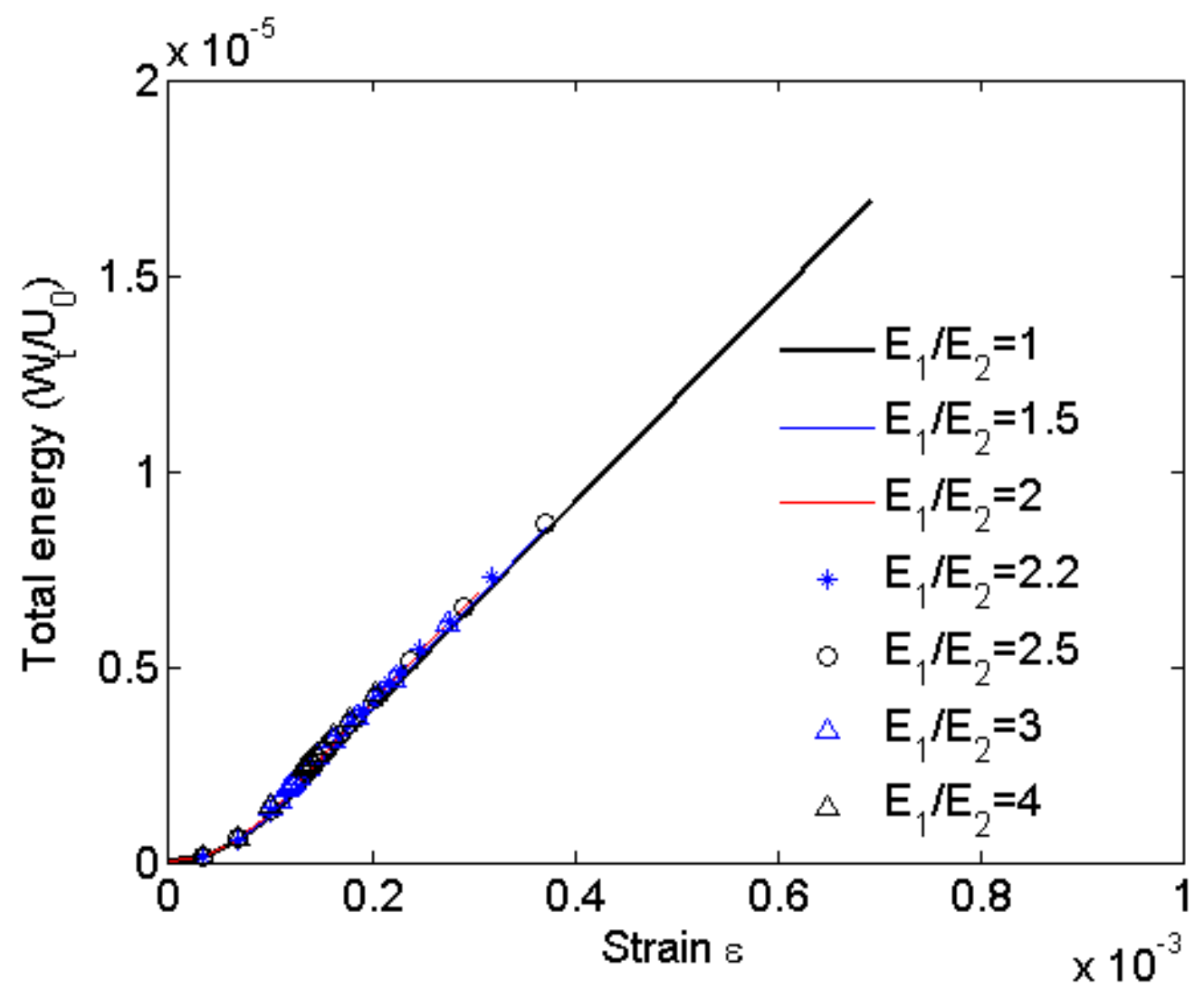

(c)

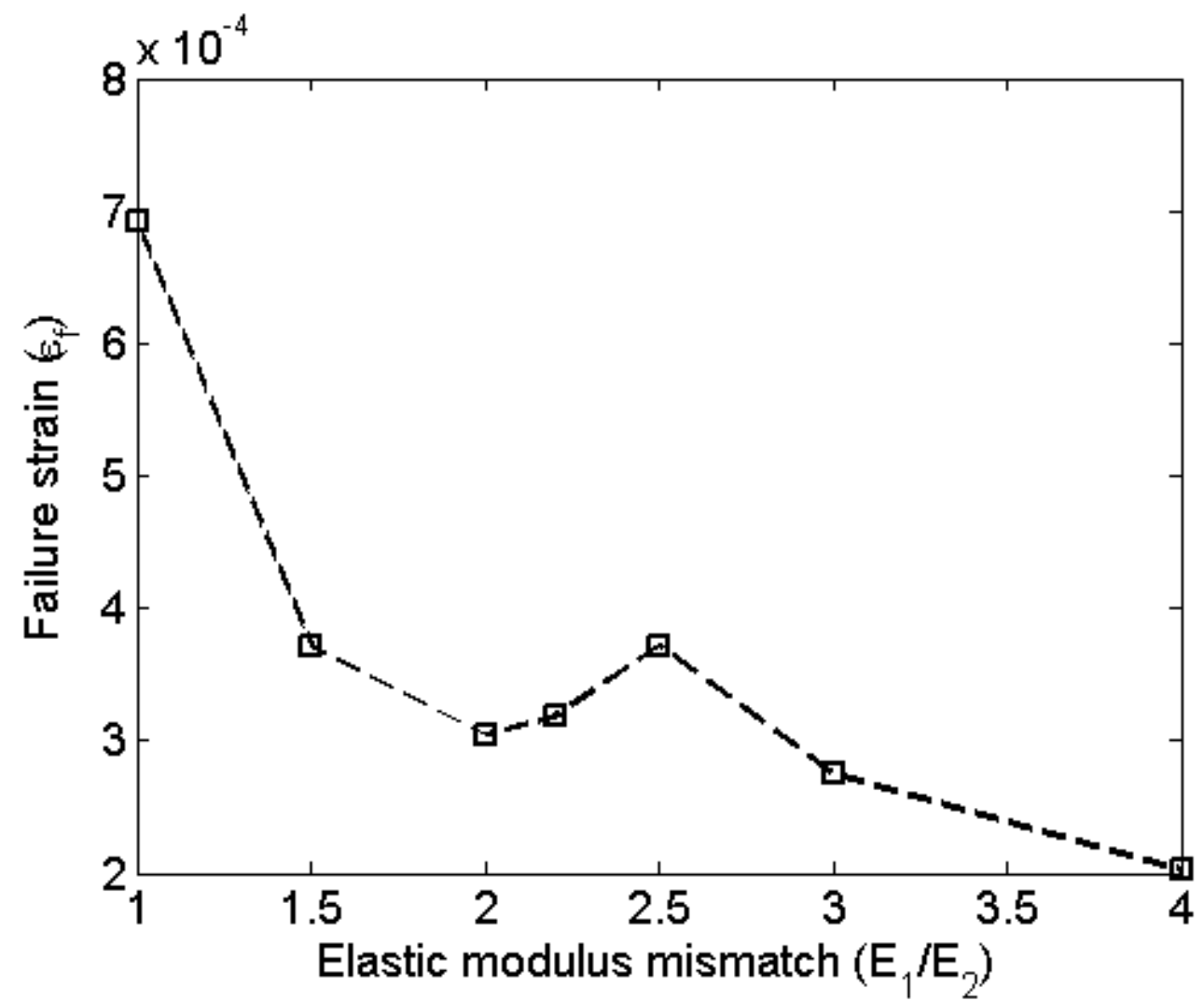


(d)

Figure 6. Effect of elastic modulus mismatch $\left(E_{1} / E_{2}\right)$ on the fracture of dentin. The materials parameters used in numerical simulations are $\sigma_{1} / \sigma_{i}=0.05, G_{l} / G_{i}=0.05$, $\delta_{c l} / \delta_{c i}=0.005, \sigma_{i} / \sigma_{2}=4, G_{i} / G_{2}=1, \delta_{c i} / \delta_{c 2}=0.4, f_{v}=0.087$ and $f_{1}=0.262$. (a) Toughness of the two-layered structure of dentin. (b) von Mises stress contour of the damaged two-layered structure of dentin. (c) The variations of total energy with overall strain. (d) The variation of failure strain with elastic modulus mismatch. 


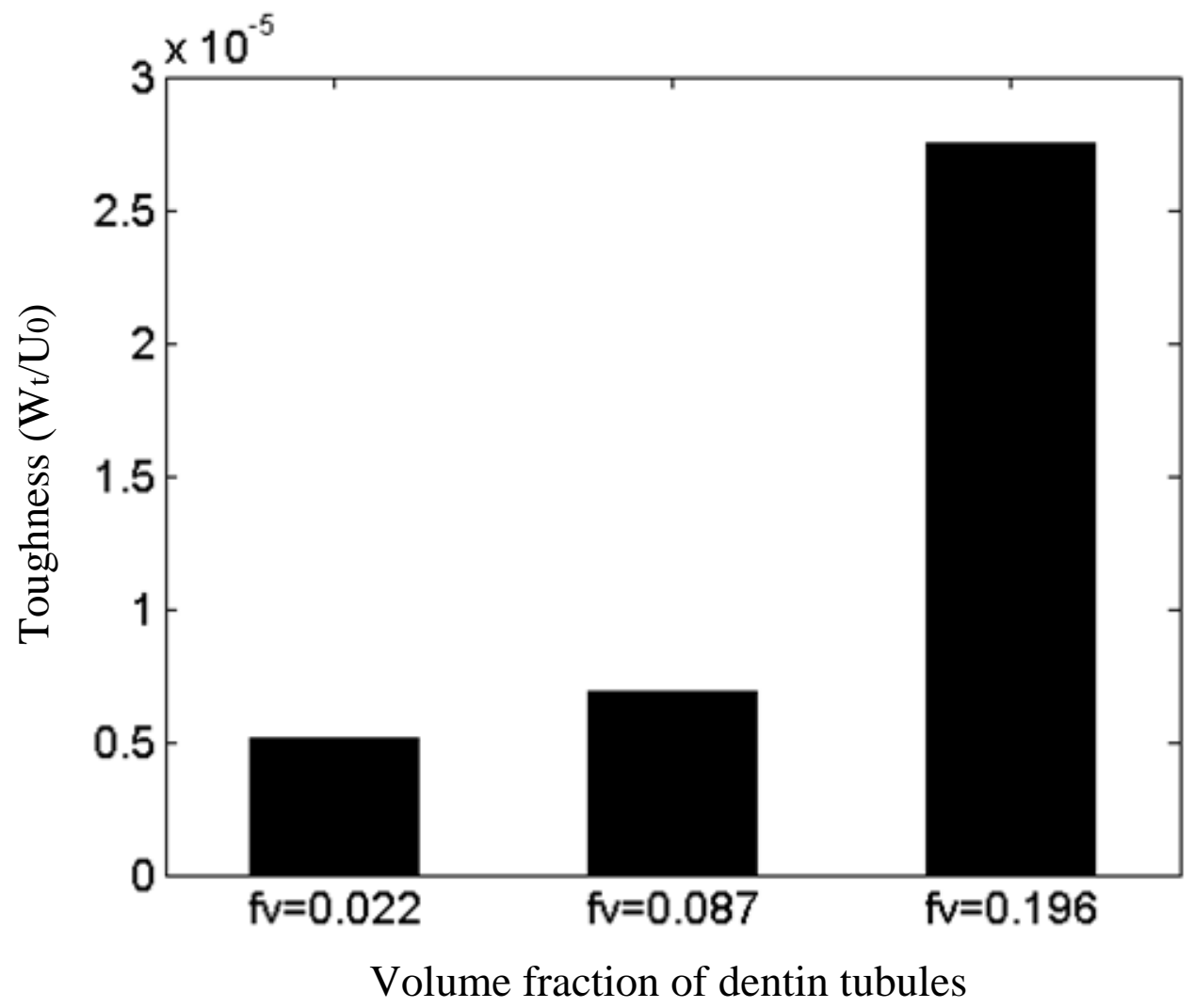

(a)

von Mises stress $\sigma_{\mathrm{q}} / \sigma_{2}$
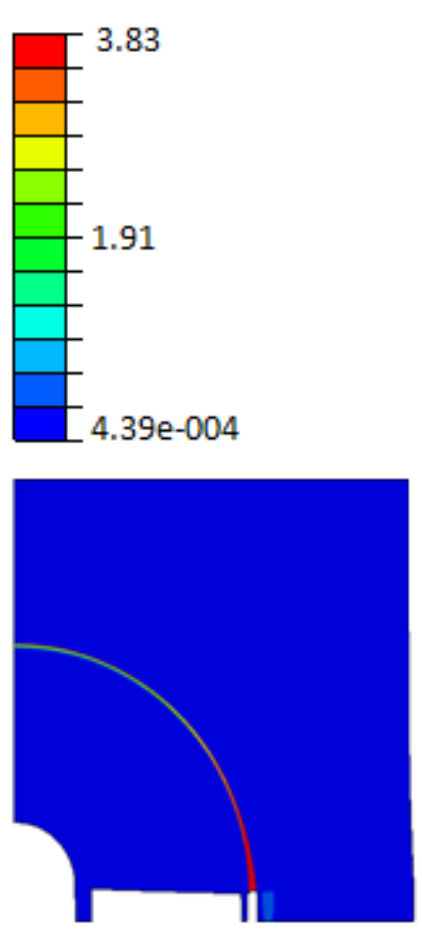

$f_{v}=0.022$
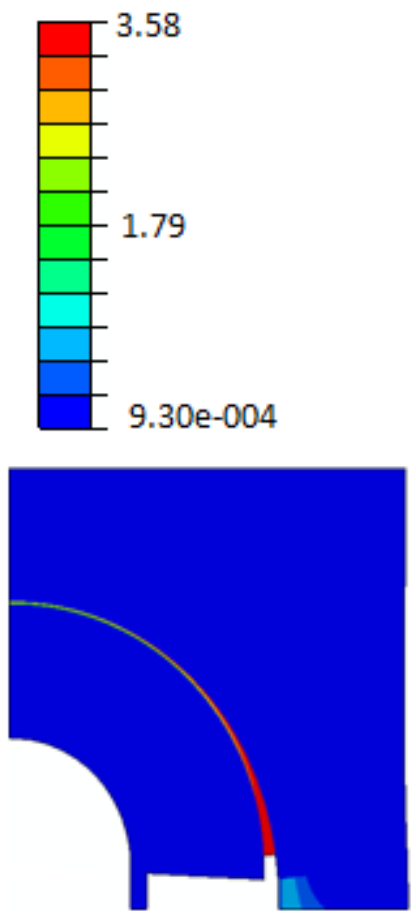

$f_{v}=0.087$
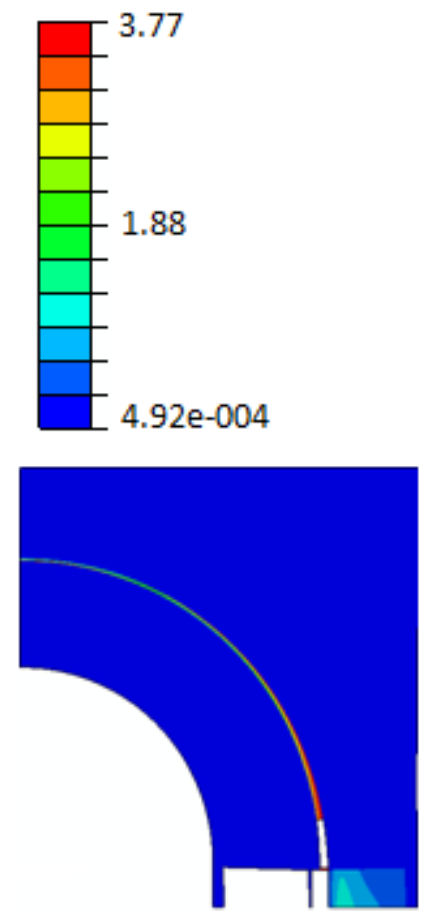

$f_{v}=0.196$ 
(b)

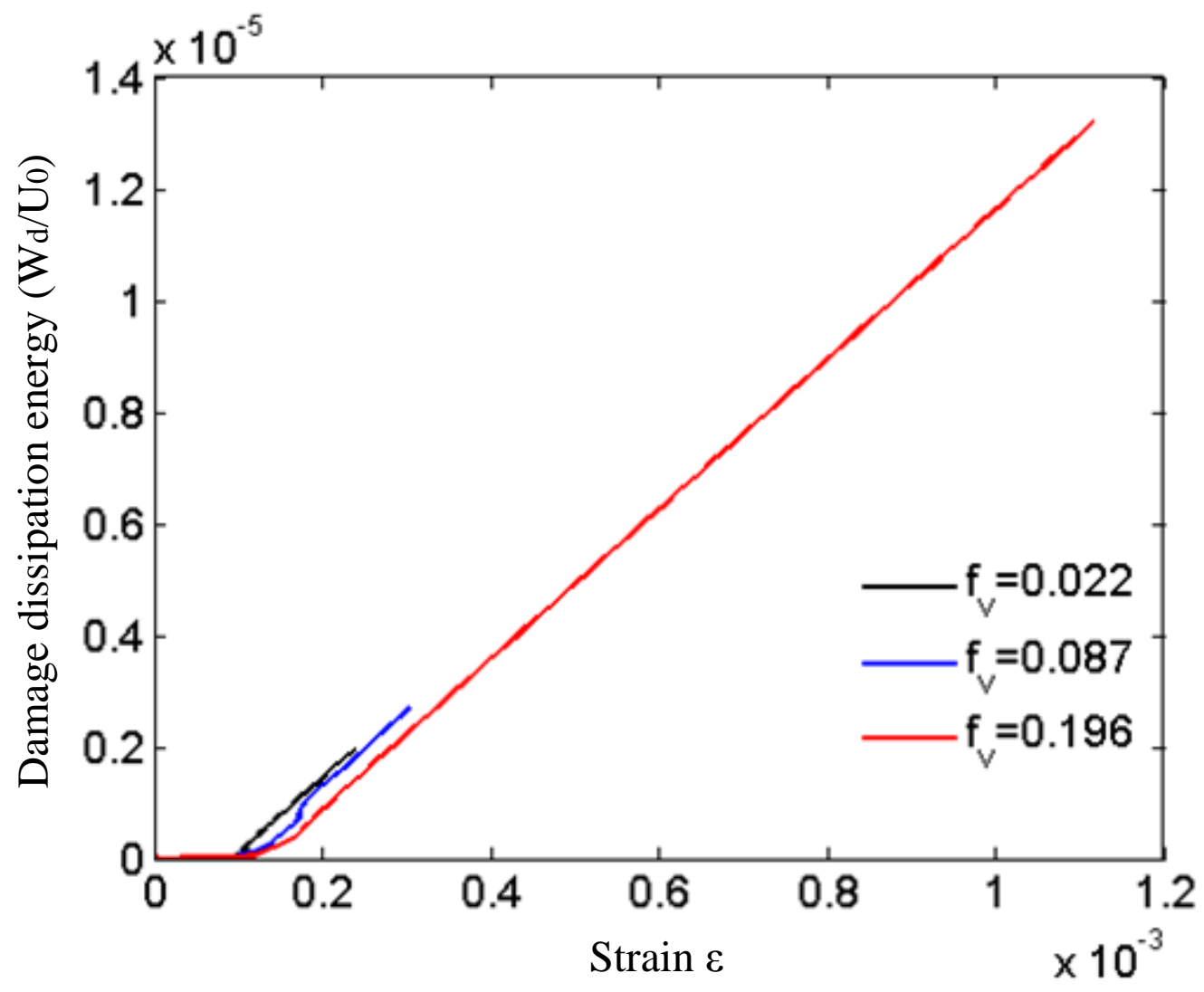

(c) 


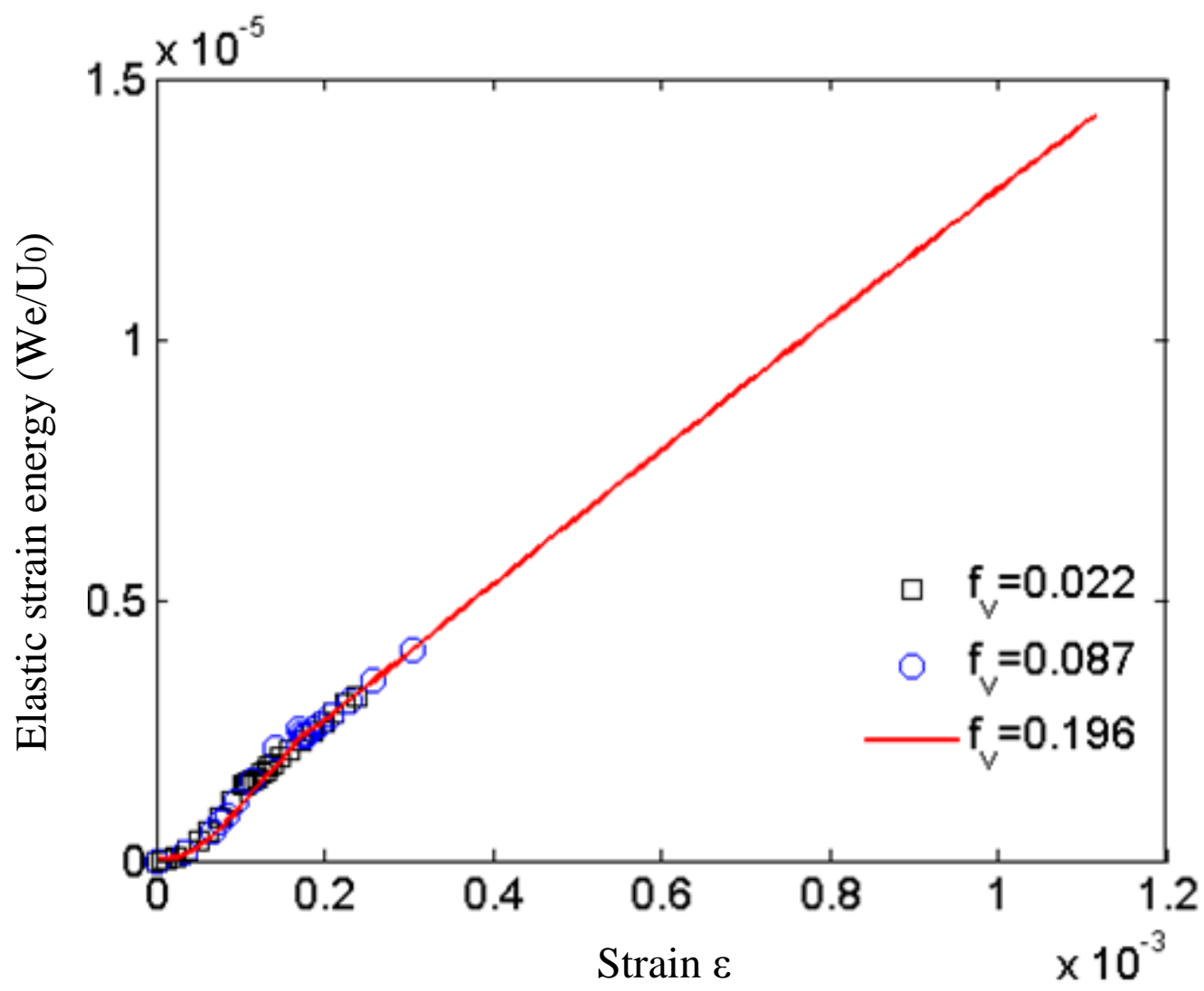

(d)

Figure 7. Effect of the volume fraction of dentin tubules on the fracture of dentin. The material parameters used in numerical simulations are $\sigma_{l} / \sigma_{i}=0.05, G_{l} / G_{i}=0.05, \delta_{c l} / \delta_{c i}$ $=0.005, \sigma_{i} / \sigma_{2}=4, G_{i} / G_{2}=1, \delta_{c i} / \delta_{c 2}=0.4, E_{1} / E_{2}=2$ and $f_{1}=0.262$. (a) Toughness of the two-layered structure of dentin. (b) von Mises stress contour of the damaged two-layered structure of dentin. (c) The variations of damage dissipation energy with overall strain. (d) The variations of elastic strain energy with overall strain. 


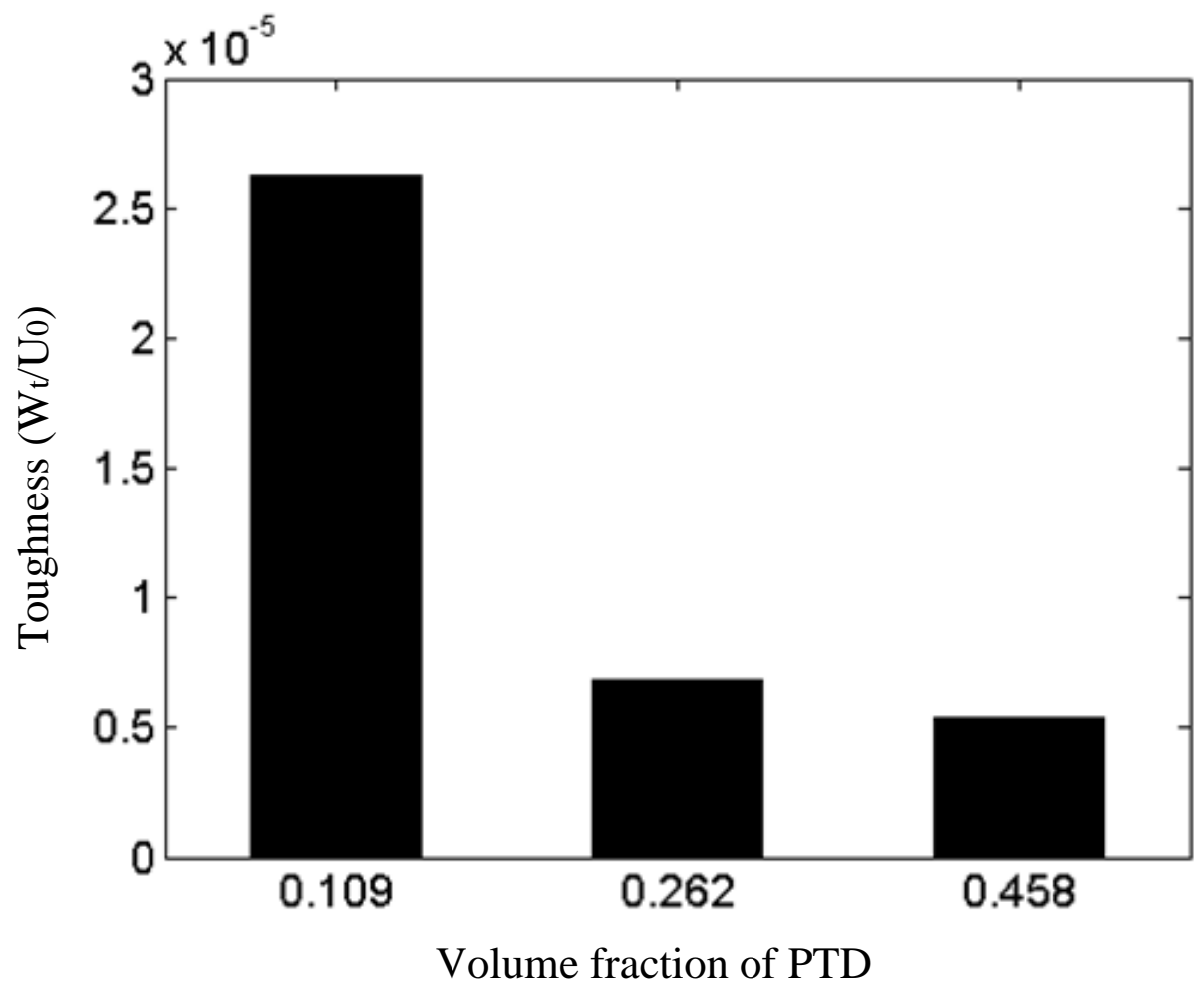

(a)

von Mises stress $\sigma_{2} / \sigma_{2}$
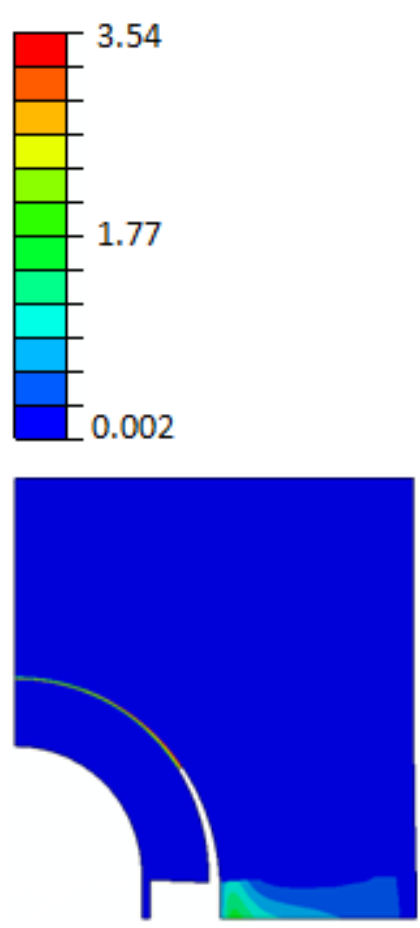

$f_{1}=0.109$
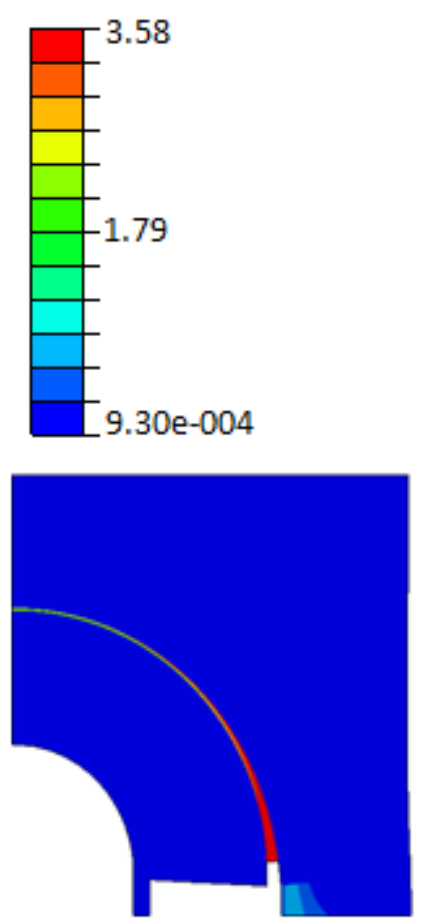

$f_{1}=0.262$
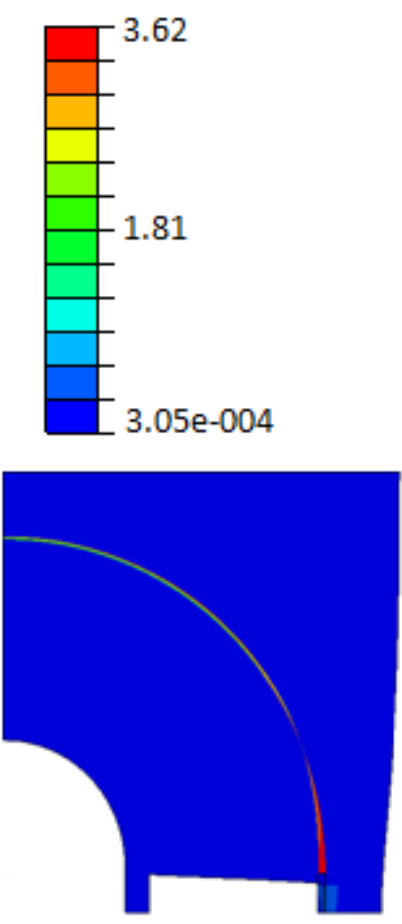

$f_{1}=0.458$ 


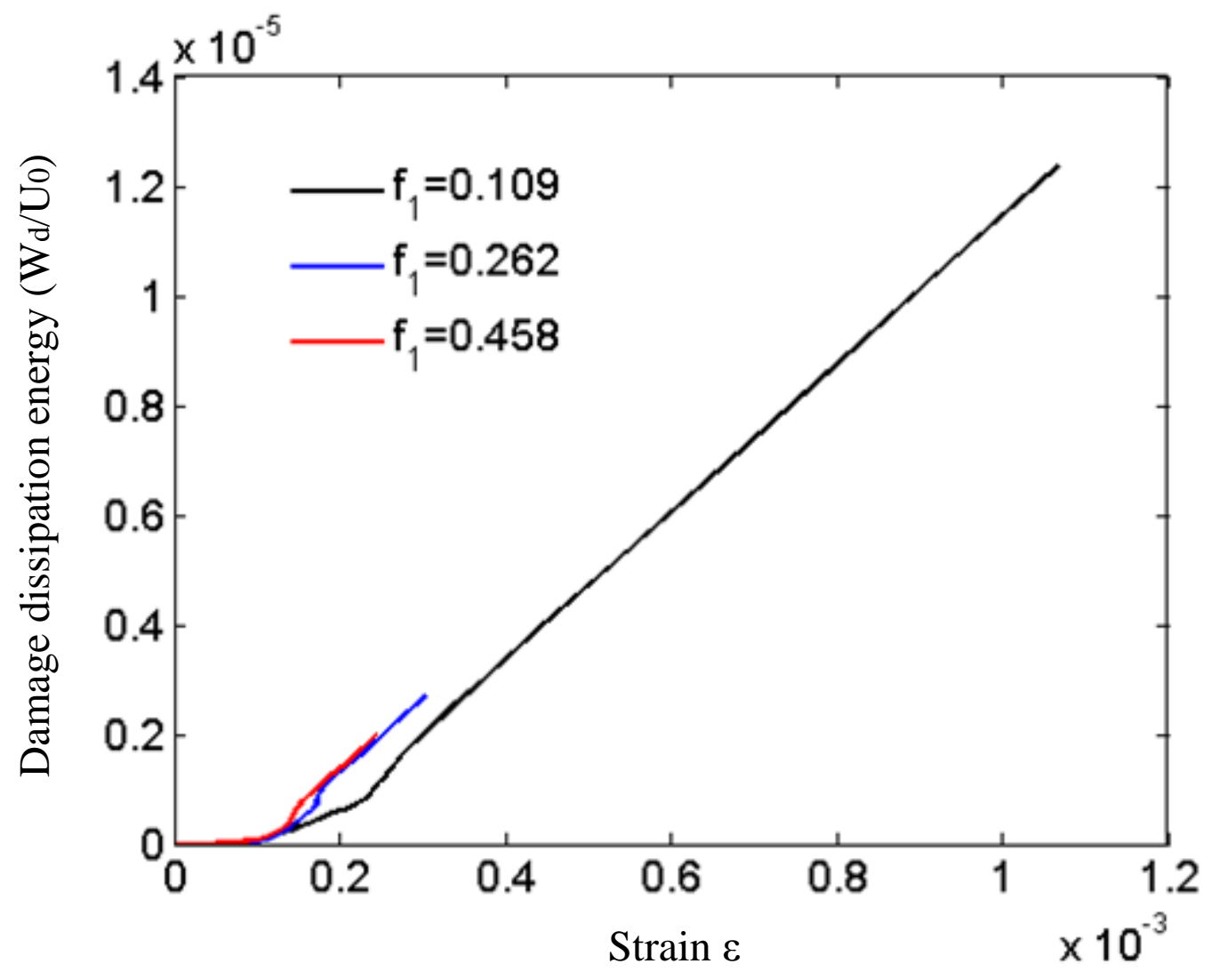

(c) 


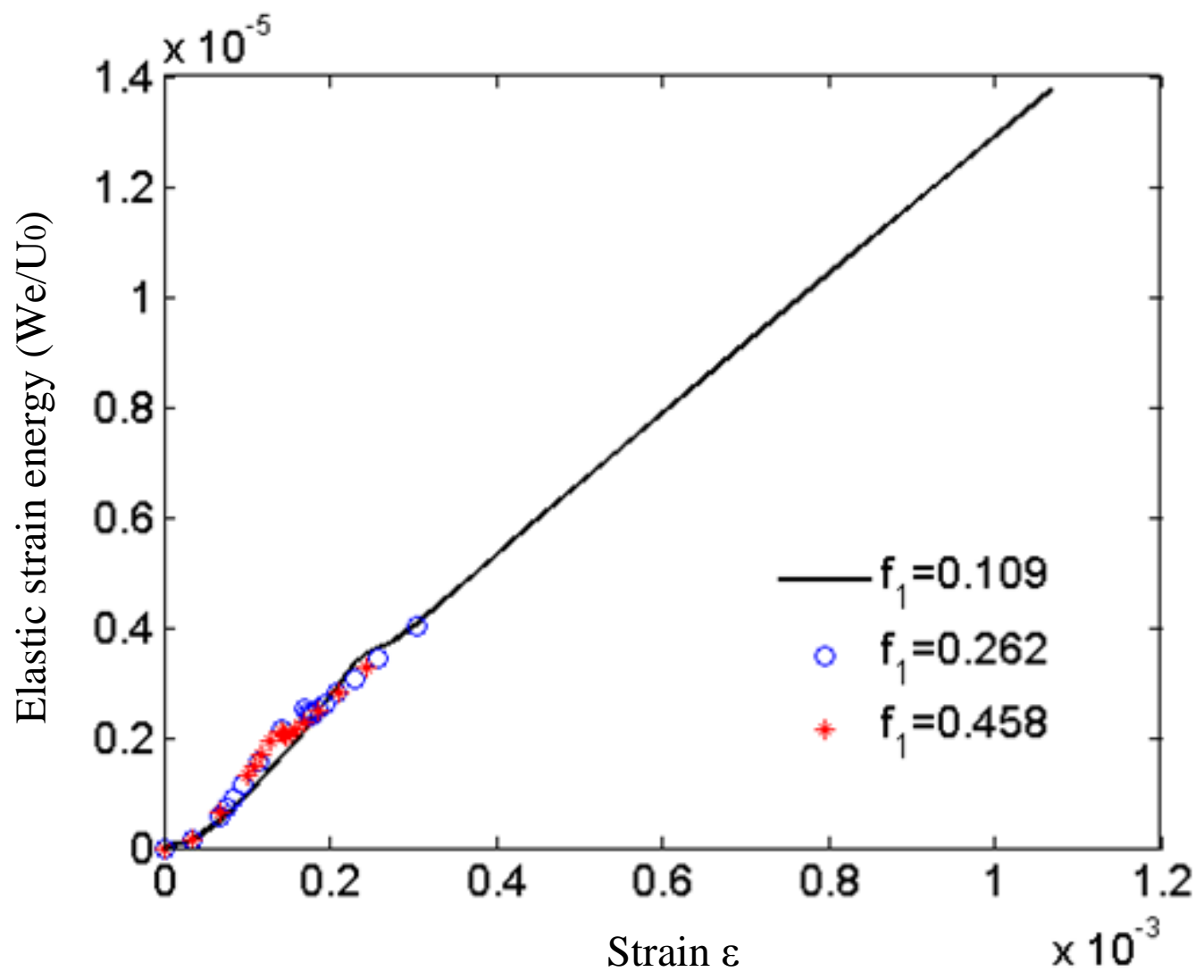

(d)

Figure 8. Effect of volume fraction of PTD on the fracture of dentin. The material parameters used in numerical simulations are $\sigma_{l} / \sigma_{i}=0.05, G_{l} / G_{i}=0.05, \delta_{c l} / \delta_{c i}=$ $0.005, \sigma_{i} / \sigma_{2}=4, G_{i} / G_{2}=1, \delta_{c i} / \delta_{c 2}=0.4, E_{1} / E_{2}=2$ and $f_{v}=0.087$. (a) Toughness of the two-layered structure of dentin. (b) von Mises stress contour of the damaged two-layered structure of dentin. (c) The variations of damage dissipation energy with overall strain. (d) The variations of elastic strain energy with overall strain. 